\title{
Existence of the global attractor to fractional order generalized coupled nonlinear Schrödinger equations with derivative
}

\author{
Wenjing Song ${ }^{1}$ and Ganshan Yang ${ }^{1,2^{*}}$
}

\section{"Correspondence: \\ ganshanyang@aliyun.com ${ }^{1}$ Department of Mathematics, Northwest University, Xi'an, China ${ }^{2}$ Department of Mathematics, Yunnan Nationalities University, Kunming, China}

\begin{abstract}
In this paper, we are concerned with the fractional Schrödinger equation with time fraction order, fractional Laplacian, and derivative terms. The existence of weak solution is established, as well as the existence of global attractor is obtained under some conditions for this equations.

MSC: 35Q41; 35B56; 35B41; 76D05

Keywords: Coupled nonlinear Schrödinger equations; Ginzburg-Landau equations; Global attractor; Fractional differential equation
\end{abstract}

\section{Introduction}

The nonlinear Schrödinger (NLS) equation provides a canonical description of envelope dynamics of quasi-monochromatic plane wave propagation processes which are negligible. The dynamics are linear within short propagation distance and short time, but cumulative nonlinear interactions lead to an important modulation of the wave amplitude on large spatial and temporal scales.

In optics, it can also be considered as the extension to nonlinear media of the paraxial approximation used for linear waves propagating in some random medium. However, by Kaminow [1], we know that single-mode optical fibers are not really single-mode, but actually bimodal according to the presence of birefringence. This birefringence can influence the way in which an optical fiber evolves during the propagation travel along the fiber. Indeed, it occurs that the linear birefringence makes a pulse split into two, and nonlinear birefringent traps them together against splitting. Menyuk [2,3] showed that the evolution of two orthogonal pulse envelopes in birefringent optical fibers can be governed by the following coupled nonlinear Schrödinger system:

$$
\begin{aligned}
& i \phi_{t}+\phi_{x x}+\left(|\phi|^{2}+e|\psi|^{2}\right) \phi=0, \\
& i \psi_{t}+\psi_{x x}+\left(e|\phi|^{2}+|\psi|^{2}\right) \psi=0,
\end{aligned}
$$

where $e$ is a positive constant depending on the anisotropy of the fibers.

(c) The Author(s) 2018. This article is distributed under the terms of the Creative Commons Attribution 4.0 International License (http://creativecommons.org/licenses/by/4.0/), which permits unrestricted use, distribution, and reproduction in any medium, provided you give appropriate credit to the original author(s) and the source, provide a link to the Creative Commons license, and indicate if changes were made. 
When $e=0$, system (1.1)-(1.2) becomes two decoupled nonlinear Schrödinger equations.

When $e=1$, system (1.1)-(1.2) is known as Manakov equations. The integrability of this system was proved by Manakov in 1974, and we shall regard it as the Integrable Manakov System (IMS).

Equations (1.1)-(1.2) are important for a number of physical applications (see [1-7]) when $e$ is positive and all the remaining constants are set equal to 1. For example, when $e=2$ for two-mode optical fibers; when $e=2 / 3$ for propagation of two modes in fibers with strong birefringence, and in the general case $2 / 3 \leq e \leq 2$ for elliptical eigenmodes. The special value $e=1$ (IMS) corresponds to at least two possible physical cases, one is the case of a purely electrostrictive nonlinearity, and another is in the elliptical birefringence case, when the angle between the major and minor axes of the birefringence ellipse is approximately $35^{\circ}$. Moreover, the experimental observation of Manakov solitons in crystals has been reported. The pulse-pulse collision between wavelength-divisionmultiplexed channels of optical fiber transmission systems are described by (1.1)-(1.2) with $e=2$ (Hasewaga and Kodama [4]).

Since the coupled nonlinear Schrödinger (CNLS) equations describe the propagation of light waves in a nonlinear birefringent optical fiber, up to now, they have been studied intensively over 30 years to realize the idea of using optical solitons as information bits in high-speed telecommunication systems (see [8-19]). Moreover, collision of solitary waves is a common phenomenon in science and engineering and it has diverse applications in many areas of physics, including nonlinear optics, plasma physics, and hydrodynamics.

Notice that generalized coupled nonlinear Ginzburg-Laudau equations are more common than GCNLS equations and are supplemented by external force:

$$
\begin{aligned}
& i u_{1 t}-(b+a i) \Delta u_{1}+g\left(\left|u_{1}\right|^{2}+\left|u_{2}\right|^{2}\right) u_{1}=f_{1}, \\
& i u_{2 t}-(b+a i) \Delta u_{2}+g\left(\left|u_{1}\right|^{2}+\left|u_{2}\right|^{2}\right) u_{2}=f_{2} .
\end{aligned}
$$

Firstly, we focus on generalized coupled nonlinear Ginzburg-Laudau equations which are more common than GCNLS equations and are supplemented with damping and external force as follows:

$$
\begin{aligned}
& i u_{1 t}-(b+a i) \Delta u_{1}+g\left(\left|u_{1}\right|^{2}+\left|u_{2}\right|^{2}\right) u_{1}+i r u_{1}=f_{1}, \\
& i u_{2 t}-(b+a i) \Delta u_{2}+g\left(\left|u_{1}\right|^{2}+\left|u_{2}\right|^{2}\right) u_{2}+i \sigma u_{2}=f_{2},
\end{aligned}
$$

where $u_{1}, u_{2}$ are the wave amplitudes in two polarizations, $a, b$ are positive real numbers, $r, \sigma>0$ are the damping parameters, $g(s)$ is a nonnegative smooth function on $R^{+}$, and the external forcing $f_{1}(x)$ and $f_{2}(x)$ are independent of $t$, belonging to $L^{2}(\Omega)$, where $\Omega$ is an open bounded set in $R^{n}$.

With the appearance of memory materials, a great attention has been focused on the study of problems involving the fractional Laplacian

$$
\begin{aligned}
& i u_{1 t}+(b+a i)(-\Delta)^{s} u_{1}+g\left(\left|u_{1}\right|^{2}+\left|u_{2}\right|^{2}\right) u_{1}+i r u_{1}=f_{1}, \\
& i u_{2 t}+(b+a i)(-\Delta)^{s} u_{2}+g\left(\left|u_{1}\right|^{2}+\left|u_{2}\right|^{2}\right) u_{2}+i \sigma u_{2}=f_{2} .
\end{aligned}
$$


Consider the initial conditions

$$
u_{1}(x, 0)=u_{10}(x), \quad u_{2}(x, 0)=u_{20}(x)
$$

and the boundary condition

$$
u_{1}(x, t)=u_{2}(x, t)=0, \quad x \in \partial \Omega .
$$

We rewrite (1.5) and (1.6) into the following form:

$$
i U_{t}+(b+a i)(-\Delta)^{s} U+g\left(|U|^{2}\right) U+i Q U=F,
$$

where

$$
U=\left(u_{1}, u_{2}\right)^{T}, \quad F=\left(f_{1}, f_{2}\right)^{T}, \quad|U|^{2}=\left|u_{1}\right|^{2}+\left|u_{2}\right|^{2}, \quad Q=\left(\begin{array}{cc}
r & 0 \\
0 & \sigma
\end{array}\right),
$$

and $U(x, t)=U$ in $C(\bar{\Omega} \times R), t \in(0, T)$. We supply (1.9) with the initial and boundary conditions

$$
U_{0}=U(x, 0) ; \quad U(x, t)=0, \quad x \in \partial \Omega .
$$

The global solution of problem (1.9)-(1.10) can hardly be got. For the case of only an equation of problem (1.9), i.e., for the 2D Ginzburg-Landau equation, we obtain some explicit periodic wave solutions using the homogeneous balance principle and general Jacobi elliptic-function method and provide a blow-up solution (see [20]). Here, let us mention that there are both similarities and differences between the Schrödinger equation and the Landau-Lifshitz equation, the Landau-Lifshitz equation is more intrinsically difficult than the Ginzburg-Landau equation (see [21-24]). If $\alpha, \beta$ are positive real constants, we change the coefficients of (1.9) and get the generalized coupled nonlinear Schrödinger equations

$$
i U_{t}+\alpha(-\Delta)^{s} U+g\left(|U|^{2}\right) U+i Q U+\beta U=F
$$

It is equipped with the same initial and boundary conditions as (1.10). With the help of the extended techniques developed by Caffarelli and Silvestre [25], some existence and nonexistence of Dirichlet problem involving the fractional Laplacian on bounded domain have been established, see Refs. [26] for example.

In this paper, we would rather switch our viewpoint to the fractional order equation $\left(E_{i g, Q, F, t}\right)$ :

$$
\left(E_{i g, Q, F, t}\right)\left\{\begin{array}{l}
D^{\alpha} U(x, t)=(b i-a)(-\Delta)^{s} U(x, t)+i g\left(|U(x, t)|^{2}\right) U(x, t) \\
\quad+c U^{2}(x, t) \overline{(-\Delta)^{\frac{s}{2}} U(x, t)}+d|U(x, t)|^{2}(-\Delta)^{\frac{s}{2}} U(x, t) \\
\quad-Q U(x, t)+i \beta U(x, t)-i F(x), \quad \text { in } \Omega_{T}, \\
U(x, t)=0, \quad \text { on } \partial \Omega_{T}, \\
U(x, 0)=\Phi(x), \quad \text { in } \Omega, \\
U_{t}(x, 0)=\Psi(x), \quad \text { in } \Omega,
\end{array}\right.
$$

in space $V=H_{0}^{s}(\Omega) \times H_{0}^{s}(\Omega)$. 
Notice that (1.9) is a special case of $\left(E_{i g, Q, F, t}\right)$. The equation $\left(E_{i g, Q, F, t}\right)$ includes the fractional Laplacian, time fractional order, and derivative terms, so it plays an important role in physics and probability in finance. We refer, for example, to [27-29] and the references therein.

As far as we know, there are few articles to study the global solution to $\left(E_{i g, Q, F, t}\right)$ with three terms at the same time: the fractional order with respect to time, space fractional order, and derivative terms. It is studied only when there is the lack of the time fractional order or the space fractional order. For the case with derivative term, the existence of global solution is an open question even in the integer order case and one-dimensional space. In this paper we first build the existence of weak solution to $\left(E_{i g, Q, F, t}\right)$, then we prove the existence of global attractor of $(1.11)$ in $L^{2}(\Omega) \times L^{2}(\Omega)$, and the dynamic motions will be given under the condition $G(\rho)=\int_{0}^{\rho} g(\tau) d \tau \leq g(\rho) \rho(\rho \geq 0)$.

\section{Functional setting}

As usual we denote the space of (classes of) square-integrable measurable complex functions on $\Omega \subset R^{n}$ by $L^{p}(\Omega)(p \geq 1) . H^{m}(\Omega) m \in N$ is the subspace of $L^{2}$-functions whose distribution derivatives of order no more than $m$ belong to $L^{2}(\Omega) . H_{0}^{1}(\Omega)$ denotes the space of functions in $H^{1}(\Omega)$ whose trace vanishes on $\partial \Omega$.

The scalar product and norm on $L^{2}(\Omega)$ are

$$
(u, v)=\int_{\Omega} u(x) \bar{v}(x) d x, \quad\|u\|=(u, u)^{1 / 2},
$$

and we set

$$
(u, v)_{m}=\sum_{[\alpha]=m}\left(D^{\alpha} u, D^{\alpha} v\right), \quad\|u\|_{m}=(u, u)_{m}^{1 / 2}
$$

where $u, v \in H^{m}(\Omega)$ and $[\alpha]=\alpha_{1}+\alpha_{2}+\cdots+\alpha_{n}$ is length of the multi-index $\alpha=$ $\left(\alpha_{1}, \alpha_{2}, \ldots, \alpha_{n}\right) \in N^{n}$.

The basic Hilbert space $L^{2}(\Omega) \times L^{2}(\Omega)$ is denoted by $H$, and we define the unbounded operator $-\Delta$ on $H$ with domain

$$
D(-\Delta)=\left(H^{2}(\Omega) \times H^{2}(\Omega)\right) \cap\left(H_{0}^{1}(\Omega) \times H_{0}^{1}(\Omega)\right)
$$

such that

$$
-\Delta U=-\sum_{i=1}^{n} \frac{\partial^{2} U}{\partial x_{i}^{2}} \in H, \quad U \in D(-\Delta) .
$$

The operator $I-\Delta$ is self-adjoint, positive on $H$, and realizes an isomorphism from $D(-\Delta)$ onto $H$. We deduce from the compactness of the embedding of $H^{1}(\Omega)$ into $L^{2}(\Omega)$ that $(I-\Delta)^{-1}$ is a compact self-adjoint operator in $H$. Thus, there exists an orthonormal Hilbert basis of $H$ consisting of eigenvectors $\omega_{j}$ of $-\Delta$ in $H_{0}^{1}(\Omega) \times H_{0}^{1}(\Omega)$, associated to the eigenvalues $\lambda_{j}$ :

$$
\begin{gathered}
-\Delta \omega_{j}=\lambda_{j} \omega_{j}, \quad\left\|\omega_{j}\right\|_{H}=1, \\
0<\lambda_{1} \leq \lambda_{2} \leq \cdots, \lambda_{j} \rightarrow \infty \text { as } j \rightarrow \infty(\text { see }[30]) .
\end{gathered}
$$


In fact, let $w_{j}$ be an orthonormal basis of $L^{2}(\Omega)$ consisting of eigenvectors $\omega_{j}$ associated to the eigenvalues $\mu_{j}$. Let

$$
\omega_{2 j-1}=\left(w_{j}, 0\right), \quad \omega_{2 j}=\left(0, w_{j}\right), \quad j=1,2, \ldots
$$

For every $U=(u, v)=\left(\sum_{j=1}^{\infty} \beta_{1 j} w_{j}, \sum_{j=1}^{\infty} \beta_{2 j} w_{j}\right)=\sum_{j=1}^{\infty} \int_{\Omega}\left(\beta_{1 j} w_{2 j-1}+\beta_{2 j} w_{2 j}\right) d x$.

If we set

$$
V=H_{0}^{1}(\Omega) \times H_{0}^{1}(\Omega)
$$

according to the boundary condition, we consider norms

$$
\begin{gathered}
\|U\|_{H}=\left(\left\|u_{1}\right\|^{2}+\left\|u_{2}\right\|^{2}\right)^{1 / 2}, \\
\|U\|_{V}=\left(\left\|u_{1}\right\|_{1}^{2}+\left\|u_{2}\right\|_{1}^{2}\right)^{1 / 2},
\end{gathered}
$$

and the scalar product can be written as

$$
\left(\left(u_{1}, u_{2}\right),\left(w_{1}, w_{2}\right)\right)=\int_{\Omega} u_{1} \bar{w}_{1}+u_{2} \bar{w}_{2} d x
$$

For every given $s>0$, we define

$$
(-\Delta)^{s} \omega_{j}=\lambda_{j}^{s} \omega_{j}
$$

if and only if

$$
\left((-\Delta)^{s} \omega_{j}, v\right)=\lambda_{j}^{s}\left(\omega_{j}, v\right), \quad v \in H_{0}^{1}
$$

for those eigenvectors $\omega_{j}$ of $-\Delta$, associated to the eigenvalues $\lambda_{j}$.

The powers $(-\Delta)^{s}, s \in R$, are well-defined and the space $H_{s}=D\left((-\Delta)^{s / 2}\right)$ and its dual space $H_{s}{ }^{\prime}=D\left((-\Delta)^{-s / 2}\right)$ are of particular interest in what follows. It should be noticed that

$$
\begin{aligned}
& H_{s}=\left\{U \mid U=\left(u_{1}, u_{2}\right)=\left(\sum_{j=1}^{\infty} \beta_{1 j} w_{j}, \sum_{j=1}^{\infty} \beta_{2 j} w_{j}\right), \sum_{j=1}^{\infty} \lambda_{j}^{s} \int_{\Omega}\left(\left|\beta_{1 j}\right|^{2}+\left|\beta_{2 j}\right|^{2}\right) d x<\infty\right\} . \\
& \|U\|_{H_{s}}=\sqrt{\sum_{j=1}^{\infty} \int_{\Omega} \lambda_{j}^{s}\left(\left|\beta_{1 j}\right|^{2}+\left|\beta_{2 j}\right|^{2}\right) d x} \\
& (U, W)_{H_{s}}=\sum_{j=1}^{\infty} \lambda_{j}^{s} \int_{\Omega}\left(\beta_{1 j} \overline{\beta_{3 j}}+\beta_{2 j} \overline{\beta_{4 j}}\right) d x
\end{aligned}
$$

where

$$
\begin{aligned}
& U=\left(u_{1}, u_{2}\right)=\left(\sum_{j=1}^{\infty} \beta_{1 j} w_{j}, \sum_{j=1}^{\infty} \beta_{2 j} w_{j}\right), \\
& W=\left(u_{3}, u_{4}\right)=\left(\sum_{j=1}^{\infty} \beta_{3 j} w_{j}, \sum_{j=1}^{\infty} \beta_{4 j} w_{j}\right) .
\end{aligned}
$$


Remark 2.1 $H_{1}=H_{0}^{1}=V, H_{2}=H_{0}^{2}, H_{-1}=H^{-1}=V^{\prime}, H_{[s]}=H_{0}^{[s]}$. When $s$ is not an integer, $H_{s}$ defined by us is slightly different from the generalized Sobolev space:

$$
\left((-\Delta)^{s} U, W\right)=\left(U,(-\Delta)^{s} W\right)=\left((-\Delta)^{s / 2} U,(-\Delta)^{s / 2} W\right)=(U, W)_{H_{s}}
$$

\section{The time-fractional equations}

Note that throughout this section the letter $\alpha$ may stand either for the parameter in Eq. (1.9) or for the order of the fractional equation when we use the notation $D^{\alpha} U$. The meaning to be chosen should be clear from the context.

In order to discuss the existence of the solution for the equation $\left(E_{i g, Q, F, t}\right)$, we need to present some basic notations, definitions, and preliminary results which will be used throughout this section. We first have the following two definitions and one lemma by Kilbas [31].

Definition 3.1 The Caputo fractional derivative of order $\alpha$ of a function $f(t), t>0$, is defined as follows:

$$
D^{\alpha} f(t)=\frac{1}{\Gamma(1-\{\alpha\})} \int_{0}^{t} \frac{1}{(t-s)^{\{\alpha\}}} f^{([\alpha]+1)} d s,
$$

where $\{\alpha\},[\alpha]$ denote the fractional and the integer part of the real number $\alpha$ respectively, and $\Gamma(\cdot)$ is the gamma function.

Definition 3.2 The Riemann-Liouville fractional integral of order $\alpha$ of a function $f(t)$, $t>0$, is defined as follows:

$$
I_{0^{+}}^{\alpha} f(t)=\frac{1}{\Gamma(\alpha)} \int_{0}^{t}(t-s)^{\alpha-1} f(s) d s,
$$

provided that the right-hand side is pointwise defined on $(0, \infty)$.

Lemma 3.3 Assume $y \in C[0, T], T>0,1<\alpha<2$, then the problem

$$
D^{\alpha} u(t)=y(t), \quad t \in[0, T]
$$

has the unique solution

$$
u(t)=u(0)+u^{\prime}(0) t+\frac{1}{\Gamma(\alpha)} \int_{0}^{t}(t-s)^{\alpha-1} y(s) d s
$$

Now we establish some results of the existence of solution for the equation $\left(E_{i g, Q, F, t}\right)$.

By Lemma 3.3, we may reduce equation $\left(E_{i g, Q, F, t}\right)$ to an equivalent integral equation as the following problem:

$$
\left(E_{i g, Q, F, t}\right)\left\{\begin{array}{l}
U(x, t)=\Phi(x)+\Psi(x) t \\
\quad+\frac{1}{\Gamma(\alpha)} \int_{0}^{t}(t-s)^{\alpha-1}\left((b i-a)(-\Delta)^{s} U(x, s)+i g\left(|U(x, s)|^{2}\right) U(x, s)\right. \\
\quad+c U^{2}(x, s)(-\Delta)^{\frac{s}{2}} U(x, s)+d|U(x, s)|^{2}(-\Delta)^{\frac{s}{2}} U(x, s) \\
\quad-Q U(x, s)+i \beta U(x, s)-i F(x)) d s, \quad \text { in } \Omega_{T}, \\
U(x, t)=0, \quad \text { on } \partial \Omega_{T} .
\end{array}\right.
$$


And we set

$$
\begin{aligned}
\Upsilon(U)= & \Phi(x)+\Psi(x) t+\frac{1}{\Gamma(\alpha)} \int_{0}^{t}(t-s)^{\alpha-1}\left((b i-a)(-\Delta)^{s} U(x, s)\right. \\
& +i g\left(|U(x, s)|^{2}\right) U(x, s)+c U^{2}(x, s) \overline{(-\Delta)^{\frac{s}{2}} U(x, s)} \\
& +d|U(x, s)|^{2}(-\Delta)^{\frac{s}{2}} U(x, s) \\
& -Q U(x, s)+i \beta U(x, s)-i F(x)) d s .
\end{aligned}
$$

Definition 3.4 We call $U \in C\left([0, T] ; H_{s}^{\prime}(\Omega) \times H_{s}^{\prime}(\Omega)\right), s \geq 1$, a weak solution of the fractional order equation $\left(E_{i g, Q, F, t}\right)$ if $\int_{\Omega}(U-\Upsilon(U)) \bar{W} d x=0, \forall t \in[0, T]$ for every $W \in H_{0}^{s}(\Omega) \times$ $H_{0}^{s}(\Omega)$.

Lemma 3.5 The operator $\Upsilon(U) \in C\left([0, T] ; H_{s}^{\prime}(\Omega) \times H_{s}^{\prime}(\Omega)\right)$ is completely continuous.

Proof Set $B=\left\{U \mid\|U\|_{H_{s} \times H_{s}} \leq M\right\}$. Put

$$
\begin{aligned}
K(U)= & (b i-a)(-\Delta)^{s} U(x, s)+i g\left(|U(x, s)|^{2}\right) U(x, s)+c U^{2}(x, s) \overline{(-\Delta)^{\frac{s}{2}} U(x, s)} \\
& +d|U(x, s)|^{2}(-\Delta)^{\frac{s}{2}} U(x, s)-Q U(x, s)+i \beta U(x, s)-i F(x) .
\end{aligned}
$$

We can rewrite

$$
\Upsilon(U)=\Phi(x)+\Psi(x) t+\frac{1}{\Gamma(\alpha)} \int_{0}^{t}(t-s)^{\alpha-1} K(U) d s
$$

For each $W \in H_{0}^{s}(\Omega) \times H_{0}^{s}(\Omega)$ and $\|W\|_{H_{0}^{s}(\Omega) \times H_{0}^{s}(\Omega)} \leq 1$, when $n \leq 3 s, 0<r \leq 3,0<q \leq$ $6, \frac{1}{r}+\frac{1}{q}=1$, and $\frac{1}{2}-\frac{s}{n} \leq \frac{1}{r}, \frac{1}{2}-\frac{s}{n} \leq \frac{1}{(2 k+1) r}$ (for example $0<r \leq \frac{5}{6}, k=2$ ), using embedding theorem and Holder's inequality, we have the following inequalities:

$$
\begin{aligned}
& \left|\left\langle(b i-a)(-\Delta)^{s} U(x, s), W\right\rangle\right| \\
& \quad=\left|\int(b i-a)(-\Delta)^{\frac{s}{2}} U(x, s)(-\Delta)^{\frac{s}{2}} \bar{W} d x\right| \\
& \quad \leq \int\left|(b i-a)(-\Delta)^{\frac{s}{2}} U(x, s)(-\Delta)^{\frac{s}{2}} \bar{W}\right| \\
& \quad \leq \sqrt{a^{2}+b^{2}}\left(\int\left|(-\Delta)^{\frac{s}{2}} U\right|^{2} d x\right)^{\frac{1}{2}}\left(\int\left|(-\Delta)^{\frac{s}{2}} W\right|^{2} d x\right)^{\frac{1}{2}} \\
& \quad \leq \sqrt{a^{2}+b^{2}}\left\|(-\Delta)^{\frac{s}{2}} U\right\|_{H}\left\|(-\Delta)^{\frac{s}{2}} W\right\|_{H}+C\|U\|_{V}^{5}\|W\|_{V} \\
& \quad \leq \sqrt{a^{2}+b^{2}}\|U\|_{H_{s}}\|W\|_{H_{s}} \\
& \quad \leq M_{1}, \\
& \left|\left\langle|U|^{2}(-\Delta)^{\frac{s}{2}} U, W\right\rangle\right| \\
& \quad \leq\left\||U|^{2}\right\|_{L^{r}}\left\|(-\Delta)^{\frac{s}{2}} U\right\|_{L^{2}}\|W\|_{L^{q}} \\
& \quad \leq\|U\|_{H_{s}}^{3} \\
& \quad \leq M M_{2}
\end{aligned}
$$




$$
\begin{aligned}
& \left.|| g\left(|U|^{2} U, W\right)\right\rangle \mid \\
& \quad \leq\left\|g\left(|U|^{2}\right) U\right\|_{L^{r}}\|W\|_{L^{p}} \\
& \quad \leq\left(C_{0}+|U|^{2}\right)^{k r}|U|^{r}\|W\|_{L^{p}} \\
& \quad \leq C_{1}\left(C_{0}|U|^{r}+|U|^{2 k r+r}\right)\|W\|_{L^{p}} \\
& \quad \leq M_{3}, \\
& \quad|\langle U(x, s), W\rangle| \\
& \quad \leq\left(\int|U|^{2} d x\right)^{\frac{1}{2}}\left(\int|W|^{2} d x\right)^{\frac{1}{2}} \\
& \quad \leq\|U\|_{H}\|W\|_{H_{S}}+\|F\|_{H}\|W\|_{H_{S}} \\
& \quad \leq M_{4} .
\end{aligned}
$$

Similarly, $\left|\left\langle U^{2}(x, s)(-\Delta)^{\frac{s}{2}} \overline{U(x, s)}, W\right\rangle\right| \leq M_{5},|\langle F, W\rangle| \leq M_{6}$.

Applying the equalities above, we immediately get $|\langle K(U), W\rangle| \leq M$.

Thus, by Cauchy-Schwarz inequalities, we obtain

$$
\begin{aligned}
& \|\Upsilon(U)\|_{H_{s}^{\prime}} \\
& =\sup _{\|W\|_{H_{0}^{s}} \leq 1}|\langle\Upsilon(U), W\rangle| \\
& =\sup _{\|W\|_{H_{0}^{s}} \leq 1}\left|\langle\Phi(x), W\rangle+\langle\Psi(x), W\rangle t+\frac{1}{\Gamma(\alpha)} \int_{0}^{t}(t-s)^{\alpha-1}\langle K(U), W\rangle d s\right| \\
& \leq|||\Phi(x), W\rangle|+||\Psi(x), W\rangle t|+| \frac{1}{\Gamma(\alpha)} \int_{0}^{t}(t-s)^{\alpha-1}\langle K(U), W\rangle d s \mid \\
& \leq\|\Phi(x)\|_{L^{\infty}(\Omega)}\|W\|_{H_{s}}+\|\Psi(x)\|_{L^{\infty}(\Omega)}\|W\|_{H_{s}} T \\
& \quad+|\langle K(U), W\rangle|\left|\frac{1}{\Gamma(\alpha)} \int_{0}^{t}(t-s)^{\alpha-1} d s\right| \\
& \leq\|\Phi(x)\|_{L^{\infty}(\Omega)}+\|\Psi(x)\|_{L^{\infty}(\Omega)} T+\frac{M}{\Gamma(\alpha)}\left|\int_{0}^{t}(t-s)^{\alpha-1} d s\right| \\
& \leq\|\Phi(x)\|_{L^{\infty}(\Omega)}+\|\Psi(x)\|_{L^{\infty}(\Omega)} T-\frac{M}{\alpha \Gamma(\alpha)} t^{\alpha} \\
& \leq\|\Phi(x)\|_{L^{\infty}(\Omega)}+\|\Psi(x)\|_{L^{\infty}(\Omega)} T+\frac{M}{\alpha \Gamma(\alpha)} T^{\alpha} .
\end{aligned}
$$

Hence, $\Upsilon(U)$ is uniformly bounded.

On the other hand, given $\epsilon>0$, set

$$
\theta=\left\{\left(\|\psi(x)\|_{L^{\infty}(\Omega)}+\frac{M}{\Gamma(\alpha)}\right)^{-1} \epsilon\right\}^{\frac{1}{\alpha}} .
$$


Then, for every $W \in V, t_{1}<t_{2}, t_{1}, t_{2} \in[0, T]$, and $t_{2}-t_{1}<\theta$, one has $\left\|\Upsilon U\left(t_{2}\right)-\Upsilon U\left(t_{1}\right)\right\|_{H_{s}^{\prime}}=$ $\sup _{\|W\|_{H_{0}^{s}} \leq 1}\left|\left\langle\Upsilon U\left(t_{2}\right)-\Upsilon U\left(t_{1}\right), W\right\rangle\right| \leq \epsilon$. That is to say, $\Upsilon(U)$ is equicontinuity. In fact,

$$
\begin{aligned}
\left\|\Upsilon U\left(t_{2}\right)-\Upsilon U\left(t_{1}\right)\right\|_{H_{s}^{\prime}} & \\
= & \sup _{\|W\|_{H_{0}^{s} \leq 1}}\left|\left\langle\Upsilon U\left(t_{2}\right)-\Upsilon U\left(t_{1}\right), W\right\rangle\right| \\
= & \sup _{\|W\|_{H_{0}^{s} \leq 1}}|\langle\Psi(x), W\rangle|\left(t_{2}-t_{1}\right)+\frac{1}{\Gamma(\alpha)} \int_{0}^{t_{2}}\left(t_{2}-s\right)^{\alpha-1}|K(U), W\rangle d s \\
& -\frac{1}{\Gamma(\alpha)} \int_{0}^{t_{1}}\left(t_{1}-s\right)^{\alpha-1}\langle K(U), W\rangle d s \\
\leq & \|\Psi(x)\|_{L^{\infty}(\Omega)}\|W\|_{H_{s}}\left|t_{2}-t_{1}\right|+\frac{1}{\Gamma(\alpha)}|\langle K(U), W\rangle|\left|\int_{t_{1}}^{t_{2}}\right|\left(t_{2}-s\right)^{\alpha-1} d s|| \\
& +\left|\frac{1}{\Gamma(\alpha)} \int_{0}^{t_{1}}\right|\langle K(U), W\rangle||\left(t_{2}-s\right)^{\alpha-1}-\left(t_{1}-s\right)^{\alpha-1} d s|| \\
\leq & \|\Psi(x)\|_{L^{\infty}(\Omega)}\left|t_{2}-t_{1}\right|+\frac{M}{-\alpha \Gamma(\alpha)} t_{2}^{\alpha}-\frac{M}{-\alpha \Gamma(\alpha)} t_{1}^{\alpha} \\
= & \|\Psi(x)\|_{L^{\infty}(\Omega)}\left|t_{2}-t_{1}\right|-\frac{M}{\alpha \Gamma(\alpha)}\left(t_{2}^{\alpha}-t_{1}^{\alpha}\right) \\
\leq & \|\Psi(x)\|_{L^{\infty}(\Omega)}\left|t_{2}-t_{1}\right|+\frac{M}{\alpha \Gamma(\alpha)}\left(t_{2}^{\alpha}-t_{1}^{\alpha}\right) .
\end{aligned}
$$

In the following, we divide the proof into two cases.

Case 1: $\theta \leq t_{1}<t_{2}<T$, since $1<\alpha<2$, we get

$$
\begin{aligned}
\left\|\Upsilon U\left(t_{2}\right)-\Upsilon U\left(t_{1}\right)\right\|_{H_{s}^{\prime}} & =\sup _{\|W\|_{H_{0}^{s}} \leq 1}\left|\left\langle\Upsilon U\left(t_{2}\right)-\Upsilon U\left(t_{1}\right), W\right\rangle\right| \\
& \leq\|\Psi(x)\|_{L^{\infty}(\Omega)}\left|t_{2}-t_{1}\right|+\frac{M}{\alpha \Gamma(\alpha)}\left(t_{2}^{\alpha}-t_{1}^{\alpha}\right) \\
& =\|\Psi(x)\|_{L^{\infty}(\Omega)}\left|t_{2}-t_{1}\right|+\frac{M}{\alpha \Gamma(\alpha)} \alpha t^{\alpha-1}\left(t_{2}-t_{1}\right) \\
& \leq\|\Psi(x)\|_{L^{\infty}(\Omega)}\left|t_{2}-t_{1}\right|+\frac{M}{\Gamma(\alpha) \theta^{1-\alpha}}\left(t_{2}-t_{1}\right) \\
& =\|\Psi(x)\|_{L^{\infty}(\Omega)} \theta+\frac{M}{\Gamma(\alpha)} \theta^{\alpha} \\
& \leq\|\Psi(x)\|_{L^{\infty}(\Omega)} \theta^{\alpha}+\frac{M}{\Gamma(\alpha)} \theta^{\alpha} \\
& =\left(\|\Psi(x)\|_{L^{\infty}(\Omega)}+\frac{M}{\Gamma(\alpha)}\right) \theta^{\alpha} \leq \epsilon .
\end{aligned}
$$

Case 2: $0 \leq t_{1}, t_{2}<\alpha^{\frac{1}{\alpha}} \theta$,

$$
\begin{aligned}
\left\|\Upsilon U\left(t_{2}\right)-\Upsilon U\left(t_{1}\right)\right\|_{H_{s}^{\prime}} & =\sup _{\|W\|_{H_{0}^{s} \leq 1}}\left|\left\langle\Upsilon U\left(t_{2}\right)-\Upsilon U\left(t_{1}\right), W\right\rangle\right| \\
& \leq\|\Psi(x)\|_{L^{\infty}(\Omega)}\left|t_{2}-t_{1}\right|+\frac{M}{\alpha \Gamma(\alpha)}\left(t_{2}^{\alpha}-t_{1}^{\alpha}\right)
\end{aligned}
$$




$$
\begin{aligned}
& \leq\|\Psi(x)\|_{L^{\infty}(\Omega)} \theta+\frac{M}{\alpha \Gamma(\alpha)}\left(\alpha^{\frac{1}{\alpha}} \theta\right)^{\alpha} \\
& \leq\|\Psi(x)\|_{L^{\infty}(\Omega)} \theta^{\alpha}+\frac{M}{\Gamma(\alpha)} \theta^{\alpha} \\
& =\left(\|\Psi(x)\|_{L^{\infty}(\Omega)}+\frac{M}{\Gamma(\alpha)}\right) \theta^{\alpha} \leq \epsilon .
\end{aligned}
$$

By applying the Arzela-Ascoli theorem, we know that $\Upsilon(U): H_{s}(\Omega) \times H_{s}(\Omega) \rightarrow H_{s}^{\prime}(\Omega) \times$ $H_{s}^{\prime}(\Omega)$ is completely continuous. This completes the proof.

By Lemma 3.5, we know that $\int_{\Omega}(U-\Upsilon(U)) W d x=0, \forall t \in[0, T]$ for every $W \in H_{0}^{s}(\Omega) \times$ $H_{0}^{s}(\Omega)$. That is to say, the fractional order equation $\left(E_{i g, Q, F, t}\right)$ has a unique weak solution $U \in C\left([0, T] ; H_{s}^{\prime}(\Omega) \times H_{s}^{\prime}(\Omega)\right)$.

\section{Estimate to $U_{t}$}

For $U \in B$, from Sect. 3, we have

$$
\begin{aligned}
& \left\|U_{t}\right\|_{H_{s}^{\prime}}=\sup _{\|W\|_{H_{0}^{s}} \leq 1}\left|\left\langle U_{t}, W\right\rangle\right| \\
& =\sup _{\|W\|_{H_{0}^{s}} \leq 1}\left|\langle\Psi(x), W\rangle+\frac{1}{\Gamma(\alpha-1)} \int_{0}^{t}(t-s)^{\alpha-2}\langle K(U), W\rangle d s\right| \\
& \leq \sup _{\|W\|_{H_{0}^{s}} \leq 1}|\langle\Psi(x), W\rangle|+\left|\frac{1}{\Gamma(\alpha-1)} \int_{0}^{t}(t-s)^{\alpha-2} \sup _{\|W\|_{H_{0}^{s}} \leq 1}\langle K(U), W\rangle d s\right| \\
& \leq\|\Psi(x)\|_{L^{\infty}(\Omega)}\|W\|_{H_{0}^{s}} \\
& +\sup _{\|W\|_{H_{0}^{s}} \leq 1}|\langle K(U), W\rangle|\left|\frac{1}{\Gamma(\alpha-1)} \int_{0}^{t}(t-s)^{\alpha-2} d s\right| \\
& \leq\|\Psi(x)\|_{L^{\infty}(\Omega)}+\frac{M}{\Gamma(\alpha-1)}\left|\int_{0}^{t}(t-s)^{\alpha-2} d s\right| \\
& \leq\|\Psi(x)\|_{L^{\infty}(\Omega)}+\frac{M}{\Gamma(\alpha)} t^{\alpha-1} \\
& \leq\|\Psi(x)\|_{L^{\infty}(\Omega)}+\frac{M}{\Gamma(\alpha)} T^{\alpha-1} .
\end{aligned}
$$

Hence, $\left\|U_{t}\right\|_{H_{s}^{\prime}}$ is bounded.

Because

$$
\begin{aligned}
& \int_{0}^{t}(t-s)^{\alpha-1}(-\Delta)^{s} U(x, s) d s \\
& \quad=U(x, t)-\Phi(x)-\Psi(x) t-\frac{1}{\Gamma(\alpha)} \int_{0}^{t}(t-s)^{\alpha-1}\left(K(U)+(-\Delta)^{s} U(x, s)\right) d s,
\end{aligned}
$$

so

$$
\begin{aligned}
\int_{0}^{t}(t-s)^{\alpha-1}\left((-\Delta)^{s} U(x, s), W\right) d s= & (U(x, t), W)-(\Phi(x), W)-(\Psi(x) t, W) \\
& -\frac{1}{\Gamma(\alpha)} \int_{0}^{t}(t-s)^{\alpha-1}\left(K(U)+(-\Delta)^{s} U(x, s), W\right) d s .
\end{aligned}
$$


Integrating by parts, we have

$$
\begin{aligned}
& \int_{0}^{t}(t-s)^{\alpha-1}\left\|(-\Delta)^{\frac{s}{2}} U(x, s)\right\|_{H_{s}^{\prime}} d s \\
& =\int_{0}^{t}(t-s)^{\alpha-1} \sup _{\|W\|_{H^{s}} \leq 1}\left|\left\langle(-\Delta)^{\frac{s}{2}} U,(-\Delta)^{\frac{s}{2}} W\right\rangle\right| d s \\
& =\int_{0}^{t}(t-s)^{\alpha-1}\left|\left((-\Delta)^{s} U(x, s), W\right)\right| d s \\
& =\mid(U(x, t), W) \\
& -(\Phi(x), W)-(\Psi(x) t, W)-\frac{1}{\Gamma(\alpha)} \int_{0}^{t}(t-s)^{\alpha-1}(K(U)+\Delta U(x, s), W) d s \mid \\
& \leq|(U(x, t), W)|+\sup _{\|W\|_{H_{0}^{1}} \leq 1} \mid\langle\Phi(x), W\rangle+\langle\Psi(x), W\rangle t \\
& +\frac{1}{\Gamma(\alpha)} \int_{0}^{t}(t-s)^{\alpha-1}\left\langle K(U)+(-\Delta)^{s} U(x, s), W\right\rangle d s \mid \\
& \leq\|U\|_{H_{s}^{\prime}}+|\langle\Phi(x), W\rangle|+|\langle\Psi(x), W\rangle t| \\
& +\left|\frac{1}{\Gamma(\alpha)} \int_{0}^{t}(t-s)^{\alpha-1}\left\langle K(U)+(-\Delta)^{s} U(x, s), W\right\rangle d s\right| \\
& \leq\|U\|_{H_{s}^{\prime}}+\|\Phi(x)\|_{L^{\infty}(\Omega)}\|W\|_{H_{0}^{s}}+\|\Psi(x)\|_{L^{\infty}(\Omega)}\|W\|_{H_{0}^{1}} T \\
& +\left|\left\langle K(U)\|U\|_{H^{-1}}+, W\right\rangle\right|\left|\frac{1}{\Gamma(\alpha)} \int_{0}^{t}(t-s)^{\alpha-1} d s\right| \\
& \leq\|U\|_{H_{s}^{\prime}}+\|\Phi(x)\|_{L^{\infty}(\Omega)}+\|\Psi(x)\|_{L^{\infty}(\Omega)} T+\frac{M}{\Gamma(\alpha)}\left|\int_{0}^{t}(t-s)^{\alpha-1} d s\right| \\
& \leq\|U\|_{H_{s}^{\prime}}+\|\Phi(x)\|_{L^{\infty}(\Omega)}+\|\Psi(x)\|_{L^{\infty}(\Omega)} T-\frac{M}{\alpha \Gamma(\alpha)} t^{\alpha} \\
& \leq\|U\|_{H_{s}^{\prime}}+\|\Phi(x)\|_{L^{\infty}(\Omega)}+\|\Psi(x)\|_{L^{\infty}(\Omega)} T+\frac{M}{\alpha \Gamma(\alpha)} T^{\alpha} .
\end{aligned}
$$

Because $\langle K(U), W\rangle$ is bounded, thus from Sect. 2 we deduce $\left\langle K(U)+(-\Delta)^{s} U(x, s)\right.$, $W\rangle \leq M$. Hence, $\int_{0}^{t}(t-s)^{\alpha-1}\left\|(-\Delta)^{\frac{s}{2}} U(x, s)\right\|_{H_{s}^{\prime}} d s$ is bounded.

\section{A priori estimate of (1.9)-(1.10)}

Lemma 5.1 If $F \in H$, there is a priori estimate about solution $U(x, t) \in H^{s}$ for problems (1.9)-(1.10) as follows:

$$
\begin{aligned}
& \|U(t)\|_{H}^{2} \leq\left\|U_{0}\right\| F_{H}^{2} e^{-\gamma t}+\frac{M_{1}}{\gamma^{2}}\left(1-e^{-\gamma t}\right), \\
& \left\|(-\Delta)^{\frac{s}{2}} U\right\|_{H}^{2}+\int_{\Omega} g\left(|U|^{2}\right)|U|^{2} d x \leq C\|F\|_{H}^{2} .
\end{aligned}
$$

Proof Multiplying (1.9) by $\bar{U}$ and integrating on $\Omega$, we have

$$
i\left(U_{t}, U\right)+(b+a i)\left((-\Delta)^{s} U, U\right)+\left(g\left(|U|^{2}\right) U, U\right)+i(Q U, U)+i \beta(U, U)=(F, U) .
$$


From our definition

$$
\int_{\Omega}(-\Delta)^{s} U \bar{U} d x=\int_{\Omega}\left|(-\Delta)^{\frac{s}{2}} U\right|^{2} d x .
$$

In formula (5.1), we know that

$$
(Q U, \bar{U})=\int_{\Omega}\left(r u_{1} \bar{u}_{1}+\sigma u_{2} \bar{u}_{2}\right) d x=r\left\|u_{1}\right\|^{2}+\sigma\left\|u_{2}\right\|^{2},
$$

we choose the imaginary part of (5.1)

$$
\frac{1}{2} \frac{d}{d t}\|U\|_{H}^{2}+a\left\|(-\Delta)^{\frac{s}{2}} U\right\|_{H}^{2}+\left(r\left\|u_{1}\right\|^{2}+\sigma\left\|u_{2}\right\|^{2}\right)=\operatorname{Im}(F, \bar{U}) .
$$

Set $\gamma=\min \{r, \sigma\}$, we have

$$
\frac{1}{2} \frac{d}{d t}\|U\|_{H}^{2}+a\left\|(-\Delta)^{\frac{s}{2}} U\right\|_{H}^{2}+\gamma\|U\|_{H}^{2} \leq \operatorname{Im}(F, \bar{U}) .
$$

Because of $a\left\|(-\Delta)^{\frac{s}{2}} U\right\|_{H}^{2} \geq 0$, we get

$$
\frac{1}{2} \frac{d}{d t}\|U\|_{H}^{2}+a\left\|(-\Delta)^{\frac{s}{2}} U\right\|_{H}^{2}+\gamma\|U\|_{H}^{2} \leq \frac{\gamma}{2}\|U\|_{H}^{2}+\frac{1}{2 \gamma}\|F\|_{H}^{2} .
$$

To simplify the formula,

$$
\frac{d}{d t}\|U\|_{H}^{2}+\gamma\|U\|_{H}^{2}+2 a\left\|(-\Delta)^{\frac{s}{2}} U\right\|_{H}^{2} \leq \frac{1}{\gamma}\|F\|_{H}^{2},
$$

where $F \in H$ and $\|F\|_{H}^{2} \leq M_{1}$. By using Gronwall's inequality, we have

$$
\|U(t)\|_{H}^{2} \leq\left\|U_{0}\right\|_{H}^{2} e^{-\gamma t}+\frac{M_{1}}{\gamma^{2}}\left(1-e^{-\gamma t}\right) .
$$

Then

$$
\limsup _{t \rightarrow \infty}\|U\|_{H}^{2} \leq \frac{M_{1}}{\gamma^{2}}
$$

Deducing from (5.3), we have

$$
\frac{1}{2} \frac{d}{d t}\|U\|_{H}^{2}+a\left\|(-\Delta)^{\frac{s}{2}} U\right\|_{H}^{2} \leq \gamma\|U\|_{H}^{2}+\frac{1}{8 \gamma}\|F\|_{H}^{2} \leq \frac{M_{1}}{8 \gamma}+\frac{M_{1}}{\gamma^{2}} .
$$

We integrate the equation above for $t \in(0, T)$,

$$
\|U(T)\|_{H}^{2}+2 a \int_{0}^{T}\left\|(-\Delta)^{\frac{s}{2}} U(s)\right\|_{H}^{2} d s \leq 2 T M_{1}\left(\frac{1}{8 \gamma}+\frac{1}{\gamma^{2}}\right)+2\left\|U_{0}\right\|_{H}^{2}
$$

Because of $\|U(T)\|_{H}^{2} \geq 0$, and $\left\|U_{0}\right\|_{H}^{2}$ is bounded, therefore, $\int_{0}^{T}\left\|(-\Delta)^{\frac{s}{2}} U(s)\right\|_{H}^{2} d s$ is bounded. Then we come to meet the conclusion that the local solution for coupled nonlinear Ginzburg-Laudau equations exists in the space $H^{s}$, and $U(x, t) \in L^{2}\left(0, T ; H^{s}\right)$. 
At the same time, we choose the real part of (5.1) to find

$$
b\left\|(-\Delta)^{\frac{s}{2}} U\right\|_{H}^{2}+\int_{\Omega} g\left(|U|^{2}\right)|U|^{2} d x+\beta\|U\|_{H}^{2}=\operatorname{Re}(F, \bar{U}),
$$

and so

$$
b\left\|(-\Delta)^{\frac{s}{2}} U\right\|_{H}^{2}+\int_{\Omega} g\left(|U|^{2}\right)|U|^{2} d x \leq \frac{1}{\beta}\|F\|_{H}^{2} .
$$

This proves the assertion.

\section{Some a priori estimates of CNLS equations}

Lemma 6.1 If $F \in H$, the solution $U(x, t)$ of problems (1.10)-(1.11) has a priori estimates as follows:

$$
\|U(t)\|_{H}^{2} \leq\left\|U_{0}\right\|_{H}^{2} e^{-\gamma t}+\frac{M_{1}}{\gamma^{2}}\left(1-e^{-\gamma t}\right) .
$$

Proof Multiplying (1.11) by $\overline{U(x, t)}$ and integrating on $\Omega$, we have

$$
i\left(U_{t}, \bar{U}\right)+\alpha\left((-\Delta)^{s} U, \bar{U}\right)+\left(g\left(|U|^{2}\right) U, \bar{U}\right)+i(Q U, \bar{U})+(\beta U, \bar{U})=(F, \bar{U})
$$

Choosing an imaginary part of (6.2),

$$
\frac{1}{2} \frac{d}{d t} \int_{\Omega}|U|^{2} d x+(Q U, \bar{U})=\operatorname{Im} \int_{\Omega} F \bar{U} d x
$$

where $(Q U, \bar{U})=\left(\left(r u_{1}, \sigma u_{2}\right),\left(\bar{u}_{1}, \bar{u}_{2}\right)\right)=\int_{\Omega} r\left|u_{1}\right|^{2}+\sigma\left|u_{2}\right|^{2} d x$, we set $\gamma=\min \{r, \sigma\}$,

$$
\frac{1}{2} \frac{d}{d t}\|U\|_{H}^{2}+\gamma\|U\|_{H}^{2} \leq \operatorname{Im} \int_{\Omega} F \bar{U} d x
$$

We know that $U_{0} \in H$,

$$
\begin{aligned}
& \frac{1}{2} \frac{d}{d t}\|U\|_{H}^{2}+\gamma\|U\|_{H}^{2} \leq \int_{\Omega}|F \bar{U}| d x=\frac{\|F\|_{H}^{2}}{2 \gamma}+\frac{\gamma}{2}\|U\|_{H}^{2}, \\
& \frac{d}{d t}\|U\|_{H}^{2}+\gamma\|U\|_{H}^{2} \leq \frac{\|F\|_{H}^{2}}{\gamma} .
\end{aligned}
$$

Because of $F \in L^{\infty}(0, T ; H)$, we have $\|F\|_{H}^{2} \leq M_{1}, U(0) \in H$. By using Gronwall's inequality, we get

$$
\|U(t)\|_{H}^{2} \leq\left\|U_{0}\right\|_{H}^{2} e^{-\gamma t}+\frac{M_{1}}{\gamma^{2}}\left(1-e^{-\gamma t}\right),
$$

then we finally get

$$
\limsup _{t \rightarrow \infty}\|U\|_{H}^{2} \leq \frac{M_{1}}{\gamma^{2}}
$$


Lemma 6.2 If $F \in H \cap V$ and $G(s)=\int_{0}^{s} g(s) d s$ satisfies $G(s) \leq g(s) s(s \geq 0)$, the solution $U(x, t)$ of problems (1.10)-(1.11) has a priori estimates as follows:

$$
\eta(U) \leq \eta\left(U_{0}\right) e^{-2 \gamma t}+C_{\infty}\left(1-e^{-2 \gamma t}\right)
$$

here we introduced the functional equation

$$
\eta(U)=\beta\|U\|_{H}^{2}+\alpha\left\|(-\Delta)^{\frac{s}{2}} U\right\|_{H}^{2}+\int_{\Omega} G\left(|U|^{2}\right) d x-2 \operatorname{Re}(F, \bar{U}) .
$$

Proof Multiplying (1.11) by $\bar{U}_{t}$ and integrating on $\Omega$, we have

$$
i\left(U_{t}, U_{t}\right)+\alpha\left((-\Delta)^{s} U, U_{t}\right)+\left(g\left(|U|^{2}\right) U, U_{t}\right)+i\left(Q U, U_{t}\right)+\beta\left(U, U_{t}\right)=\left(F, U_{t}\right),
$$

where $\left(Q U, U_{t}\right)=\left(\left(r u_{1}+\sigma u_{2}\right),\left(u_{1 t}, u_{2 t}\right)\right)=\int_{\Omega} r u_{1} \bar{u}_{1 t}+\sigma u_{2} \bar{u}_{2 t} d x$, and

$$
\operatorname{Re}\left(r u_{1} \bar{u}_{1 t}+\sigma u_{2} \bar{u}_{2 t}\right)=\frac{r}{2} \frac{d}{d t}\left\|u_{1}\right\|_{H}^{2}+\frac{\sigma}{2} \frac{d}{d t}\left\|u_{2}\right\|_{H}^{2},
$$

and the real part of (6.9) is

$$
\begin{aligned}
& \operatorname{Re} \alpha\left((-\Delta)^{\frac{s}{2}} U,(-\Delta)^{\frac{s}{2}} U_{t}\right)+\operatorname{Re} \int_{\Omega} g\left(|U|^{2}\right) U \bar{U}_{t} d x \\
& \quad-\operatorname{Im} \int_{\Omega} r u_{1} \bar{u}_{1 t}+\sigma u_{2} \bar{u}_{2 t} d x+\operatorname{Re} \beta\left(U, U_{t}\right) \\
& =\operatorname{Re}\left(F, U_{t}\right) .
\end{aligned}
$$

Return to see the real part of (6.2),

$$
\operatorname{Im}\left(U, U_{t}\right)+\alpha\left\|(-\Delta)^{\frac{s}{2}} U\right\|_{H}^{2}+\int_{\Omega} g\left(|U|^{2}\right)|U|^{2} d x+\beta\|U\|_{H}^{2}=\operatorname{Re}(F, U),
$$

we have

$$
\begin{aligned}
& \operatorname{Im} \int_{\Omega} r u_{1} \bar{u}_{1 t}+\sigma u_{2} \bar{u}_{2 t} d x+\left(r \alpha\left\|(-\Delta)^{\frac{s}{2}} u_{1}\right\|_{H}^{2}+\sigma \alpha\left\|(-\Delta)^{\frac{s}{2}} u_{2}\right\|_{H}^{2}\right) \\
& \quad+\int_{\Omega} g\left(|U|^{2}\right)\left(r\left|u_{1}\right|^{2}+\sigma\left|u_{2}\right|^{2}\right) d x+\left(\beta r\left\|u_{1}\right\|_{H}^{2}+\beta \sigma\left\|u_{2}\right\|_{H}^{2}\right) \\
& =\operatorname{Re} \int_{\Omega} r \overline{f_{1}} u_{1}+\sigma \bar{f}_{2} u_{2} d x .
\end{aligned}
$$

Let us add (6.11) with (6.13), set $\gamma=\min \{r, \sigma\}$, we have

$$
\begin{aligned}
& \frac{\alpha}{2} \frac{d}{d t}\left\|(-\Delta)^{\frac{s}{2}} U\right\|_{H}^{2}+\frac{1}{2} \frac{d}{d t} \int_{\Omega} G\left(|U|^{2}\right) d x+\frac{\beta}{2} \frac{d}{d t}\|U\|_{H}^{2} \\
& \quad+\gamma\left\|(-\Delta)^{\frac{s}{2}} U\right\|_{H}^{2}+\gamma \int_{\Omega} g\left(|U|^{2}\right)|U|^{2} d x+\gamma \beta\|U\|_{H}^{2} \\
& \leq \operatorname{Re}\left(F, U_{t}\right)+\operatorname{Re} \int_{\Omega} r \overline{f_{1}} u_{1}+\sigma \overline{f_{2}} u_{2} d x .
\end{aligned}
$$


Because $f(x)$ is independent of $t$, so $\frac{d}{d t}(F \bar{U})=F \bar{U}_{t}$, and (6.14) can change into

$$
\begin{aligned}
& \frac{1}{2} \frac{d}{d t}\left\{\alpha\left\|(-\Delta)^{\frac{s}{2}} U\right\|_{H}^{2}+\int_{\Omega} G\left(|U|^{2}\right) d x+\beta\|U\|_{H}^{2}-2 \operatorname{Re}(F, U)\right\} \\
& \quad+\gamma\left\{\alpha\left\|(-\Delta)^{\frac{s}{2}} U\right\|_{H}^{2}+\int_{\Omega} G\left(|U|^{2}\right) d x+\beta\|U\|_{H}^{2}-2 \operatorname{Re}(F, U)\right\} \\
& \leq \operatorname{Re} \int_{\Omega} r \overline{f_{1}} u_{1}+\sigma \overline{f_{2}} u_{2} d x-2 \gamma \operatorname{Re}(F, U) .
\end{aligned}
$$

Set $\delta=\max \{r, \sigma\}$, rewrite (6.15) as

$$
\begin{aligned}
& \frac{1}{2} \frac{d}{d t} \eta(U)+\gamma \eta(U) \\
& \quad \leq \operatorname{Re} \operatorname{Re} \int_{\Omega} r \overline{f_{1}} u_{1}+\sigma \overline{f_{2}} u_{2} d x-2 \gamma \operatorname{Re}(F, U) \\
& \quad \leq(\delta+2 \gamma) \int_{\Omega}|F \bar{U}| d x \\
& \quad \leq \frac{\delta+2 \gamma}{2}\left(\|U\|_{H}^{2}+\|F\|_{H}^{2}\right)
\end{aligned}
$$

We know that $F \in L^{\infty}(0, T ; H), F_{t} \in L^{\infty}(0, T ; H)$, then $\left\|F_{t}\right\|_{H}^{2},\|F\|_{H}^{2}$ are bounded in $H$, as to (6.6), then

$$
\frac{d}{d t} \eta(U)+2 \gamma \eta(U) \leq 2 \gamma C_{\infty}
$$

By using Gronwall's inequality, we get

$$
\eta(U) \leq \eta\left(U_{0}\right) e^{-2 \gamma t}+C_{\infty}\left(1-e^{-2 \gamma t}\right) .
$$

Finally, we get

$$
\limsup _{t \rightarrow \infty} \eta(U) \leq C_{\infty}
$$

and

$$
\beta\|U\|_{H}^{2}+\alpha\left\|(-\Delta)^{\frac{s}{2}} U\right\|_{H}^{2}+\int_{\Omega} G\left(|U|^{2}\right) d x-2 \operatorname{Re}(F, \bar{U}) \leq C_{\infty} .
$$

Then

$$
\begin{aligned}
& \beta\|U\|_{H}^{2}+\alpha\left\|(-\Delta)^{\frac{s}{2}} U\right\|_{H}^{2}+\int_{\Omega} G\left(|U|^{2}\right) d x \\
& \leq C_{\infty}+2 \operatorname{Re}(F, \bar{U}) \\
& \leq C_{\infty}+\frac{1}{2}\left(\|F\|_{H}^{2}+\|U\|_{H}^{2}\right) \\
& \leq C_{\infty}+\frac{1}{2}\left(M_{1}+\frac{M_{1}}{\gamma^{2}}\right) .
\end{aligned}
$$


Lemma 6.3 If $g(s)$ is a slowly increasing function, $s \geq \frac{n}{2}$, then

$$
U_{t} \in L^{2}\left(O, T ; H^{s}\right) \cap C(0, \infty ; H)
$$

Proof Multiplying the derivation of (1.9), with respect to t, by $\bar{U}_{t}$ and integrating on $\Omega$, we have

$$
\begin{gathered}
i\left(U_{t} t, U_{t}\right)+(a+b i)\left((-\Delta)^{s} U_{t}, U_{t}\right)+\left(g^{\prime}\left(|U|^{2}\right)|U|_{t}^{2} U\right. \\
\left.+g\left(|U|^{2}\right) U_{t}, U_{t}\right)+i\left(Q U_{t}, U_{t}\right)+\beta\left(U_{t}, U_{t}\right)=0 .
\end{gathered}
$$

Choosing the imaginary part, we have

$$
\begin{aligned}
& \frac{1}{2} \frac{d}{d t}\left\|U_{t}\right\|_{H}^{2}+b\left\|(-\Delta)^{s} U_{t}\right\|_{H}^{2} \\
& \quad+\int g^{\prime}\left(|U|^{2}\right) \operatorname{Im}\left(U^{2}{\overline{U_{t}}}^{2}\right)+\int_{\Omega} r u_{1} \bar{u}_{1 t}+\sigma u_{2} \bar{u}_{2 t} d x=0 .
\end{aligned}
$$

If $g(s)$ is a slowly increasing function, $s \geq \frac{n}{2}$, then

$$
\begin{aligned}
\left.\left|\int_{\Omega} g^{\prime}\left(|U|^{2}\right)\right| U\right|_{t} ^{2} U \overline{U_{t}} d x \mid & \leq \int_{\Omega}|U|^{m}\left|U_{t}\right|^{2} d x \\
& \leq C_{1}\|U\|_{L^{\infty}}^{m}\left\|U_{t}\right\|_{H}^{2} \\
& \leq C\left\|U_{t}\right\|_{H}^{2} .
\end{aligned}
$$

We can deduce

$$
\frac{1}{2} \frac{d}{d t}\left\|U_{t}\right\|_{H}^{2}+b\left\|(-\Delta)^{s} U_{t}\right\|_{H}^{2} \leq C\left\|U_{t}\right\|_{H}^{2}
$$

This completes the proof.

\section{Existence of solution}

The existence of solutions of coupled nonlinear Schrödinger equations will be considered in this section. We apply the Galerkin method to prove the existence of global smooth solution for problem (1.10)-(1.11). Let $w_{j}=\left(w_{1 j}, w_{2 j}\right)^{T}$ be the normalized eigenfunction of the equation $-\Delta w_{j}+\lambda_{j} w_{j}=0$ with the Dirichlet boundary condition corresponding to eigenvalue $\lambda_{j}$, and $\left\{w_{j}(x)\right\}_{j=1}^{\infty} \in V$ forms a normalized orthogonal system of eigenfunctions.

For every $m \in N$, we denote the approximate solution $U_{m}(x, t)$ of $(1.10)-(1.11)$ by the following form:

$$
U_{m}(x, t)=\sum_{j=1}^{m} \beta_{j m}(t) w_{j}(x), \quad t \in[0, T]
$$

where $\beta_{j m}(t)(j=1,2, \ldots, m)$ are coefficient functions of variable $t \in(0, L)$. According to the Galerkin method, the coefficient $\beta_{j m}(t)$ is assumed to satisfy the following system of 
nonlinear ordinary equations of the first order:

$$
\left(i U_{m}^{\prime}+\alpha(-\Delta)^{s} U_{m}+g\left(\left|U_{m}\right|^{2}\right) U_{m}+i Q U_{m}+\beta U_{m}-F, w_{j}\right)=0,
$$

where $\left|U_{m}\right|^{2}=\left|u_{1 m}\right|^{2}+\left|u_{2 m}\right|^{2}, j=1,2, \ldots, m$, with the initial condition

$$
\left(U_{m}(x, 0), w_{j}(x)\right)=\left(U_{0}(x), w_{j}(x)\right) .
$$

It is obvious that

$$
\begin{aligned}
& \left(U_{m}^{\prime}(x, t), w_{j}(x)\right)=\beta_{j m}^{\prime}(t), \\
& \left(U_{m}(x, 0), w_{j}(x)\right)=\beta_{j m}(0),
\end{aligned}
$$

and $U_{0 j}(x)=\left(U_{0}(x), w_{j}(x)\right)(j=1,2, \ldots, m)$ are coefficients in the approximate expansion $\sum_{j=1}^{m} U_{0 j} w_{j}(x)$ of function $U_{0}(x)$.

Let us prove that (7.2) has solution about the unknown function $\beta_{j m}(t)$. By using the characteristic of normalized eigenfunction

$$
\begin{aligned}
& \left(w_{1 j}, w_{1 i}\right)=\left(w_{2 j}, w_{2 i}\right)=1, \quad i=j ; \\
& \left(w_{1 j}, w_{1 i}\right)=\left(w_{2 j}, w_{2 i}\right)_{0}=0, \quad i \neq j,
\end{aligned}
$$

from (3.2) we get

$$
\begin{aligned}
0= & \left(i \sum_{j=1}^{m} \beta_{j m}^{\prime} w_{1 j}, w_{1 j}\right)-\alpha\left(\sum_{j=1}^{m} \beta_{j m}(-\Delta)^{s} w_{1 j}, w_{1 j}\right) \\
& +\left(g\left(\left|\sum_{j=1}^{m} \beta_{j m} w_{1 j}\right|^{2}+\left|\sum_{j=1}^{m} \beta_{j m} w_{2 j}\right|^{2}\right) \sum_{j=1}^{m} \beta_{j m} w_{1 j}, w_{1 j}\right) \\
& +\left(i r \sum_{j=1}^{m} \beta_{j m} w_{1 j}, w_{1 i}\right)+\left(\beta \sum_{j=1}^{m} \beta_{j m} w_{1 j}, w_{1 j}\right)-\left(f(x), \bar{w}_{1 j}\right) \\
= & i \int_{\Omega} \beta_{j m}^{\prime} d x-\alpha \int_{\Omega} \sum_{j=1}^{m} \beta_{j m}(-\Delta)^{s} w_{1 j} \bar{w}_{1 j} d x \\
& +\int_{\Omega} g\left(\left|\sum_{j=1}^{m} \beta_{j m} w_{1 j}\right|^{2}+\left|\sum_{j=1}^{m} \beta_{j m} w_{2 j}\right|^{2}\right) \beta_{j m} d x \\
& +(i r+\beta) \int_{\Omega} \beta_{j m} d x-\int_{\Omega} f(x) \bar{w}_{1 j} d x \\
= & i|\Omega| \beta_{j m}^{\prime}+\alpha \sum_{j=1}^{m} \beta_{j m} \int_{\Omega}(-\Delta)^{\frac{s}{2}} w_{1 j}(-\Delta)^{\frac{s}{2}} \bar{w}_{1 i} d x \\
& +\beta_{j m} \int_{\Omega} g\left(\left|\sum_{j=1}^{m} \beta_{j m} w_{1 j}\right|^{2}+\left|\sum_{j=1}^{m} \beta_{j m} w_{2 j}\right|^{2}\right) d x \\
& +(i r+\beta)|\Omega| \beta_{j m}-\int_{\Omega} f_{1}(x) \bar{w}_{1 j} d x . \\
& +(x)
\end{aligned}
$$


That is,

$$
\begin{aligned}
0= & i|\Omega| \beta_{j m}^{\prime} \\
& +\alpha \sum_{j=1}^{m} \beta_{j m} \int_{\Omega}(-\Delta)^{\frac{s}{2}} w_{1 j}(-\Delta)^{\frac{s}{2}} \bar{w}_{1 i} d x \\
& +\beta_{j m} \int_{\Omega} g\left(\left|\sum_{j=1}^{m} \beta_{j m} w_{1 j}\right|^{2}+\left|\sum_{j=1}^{m} \beta_{j m} w_{2 j}\right|^{2}\right) d x \\
& +(i r+\beta)|\Omega| \beta_{j m}-\int_{\Omega} f_{1}(x) \bar{w}_{1 j} d x .
\end{aligned}
$$

And as to $w_{2 j}$, we have a similar conclusion

$$
\begin{aligned}
0= & i|\Omega| \beta_{j m}^{\prime}+\alpha \sum_{j=1}^{m} \beta_{j m} \int_{\Omega}(-\Delta)^{\frac{s}{2}} w_{2 j}(-\Delta)^{\frac{s}{2}} \bar{w}_{2 i} d x \\
& +\beta_{j m} \int_{\Omega} g\left(\left|\sum_{j=1}^{m} \beta_{j m} w_{1 j}\right|^{2}+\left|\sum_{j=1}^{m} \beta_{j m} w_{2 j}\right|^{2}\right) d x \\
& +(i r+\beta)|\Omega| \beta_{j m}-\int_{\Omega} f_{2}(x) \bar{w}_{2 i} d x .
\end{aligned}
$$

We know that (7.7) and (7.6) are the first order ordinary equations of unknown functions $\beta_{j m}, j=1,2, \ldots, m$. If (7.7) and (7.6) have common solution, it must satisfy that

$$
\begin{aligned}
& h\left(\beta_{1 m}, \beta_{2 m}, \ldots, \beta_{m m}\right) \\
& =\alpha \sum_{j=1}^{m} \beta_{j m} \int_{\Omega}(-\Delta)^{\frac{s}{2}} w_{1 j}(-\Delta)^{\frac{s}{2}} \bar{w}_{1 i} d x \\
& \quad+\beta_{j m} \int_{\Omega} g\left(\left|\sum_{j=1}^{m} \beta_{j m} w_{1 j}\right|^{2}+\left|\sum_{j=1}^{m} \beta_{j m} w_{2 j}\right|^{2}\right) d x \\
& \quad+(i r+\beta)|\Omega| \beta_{j m}-\int_{\Omega} f_{1}(x) \bar{w}_{1 j} d x .
\end{aligned}
$$

It is locally Lipschitz continuous in $H$.

We set $\theta(t)=\left(\beta_{1 m}(t), \beta_{2 m}(t), \ldots, \beta_{m m}(t)\right), \tilde{\theta}(t)=\left(\tilde{\beta}_{1 m}(t), \tilde{\beta}_{2 m}(t), \ldots, \tilde{\beta}_{m m}(t)\right)$, and Lipschitz continuous functions $h(\theta(t))$ are considered to satisfy

$$
|h(\theta(t))-h(\tilde{\theta}(t))| \leq C|\theta(t)-\tilde{\theta}(t)|
$$

Then

$$
\begin{aligned}
h(\theta(t))= & \alpha \sum_{j=1}^{m} \beta_{j m}(t) \int_{\Omega}(-\Delta)^{\frac{s}{2}} w_{1 m}(-\Delta)^{\frac{s}{2}} \bar{w}_{1 i} d x \\
& +\beta_{j m} \int_{\Omega} g\left(\left|\sum_{j=1}^{m} \beta_{j m} w_{1 j}\right|^{2}+\left|\sum_{j=1}^{m} \beta_{j m} w_{2 j}\right|^{2}\right) d x
\end{aligned}
$$




$$
\begin{aligned}
& +(i r+\beta)|\Omega| \beta_{j m}(t)-\int_{\Omega} f_{1}(x) \bar{w}_{1 i} d x \\
h(\tilde{\theta}(t))= & \alpha \sum_{j=1}^{m} \tilde{\beta}_{j m}(t) \int_{\Omega}(-\Delta)^{\frac{s}{2}} w_{1 m}(-\Delta)^{\frac{s}{2}} \bar{w}_{1 i} d x \\
& +\tilde{\beta}_{j m}(t) \int_{\Omega} g\left(\left|\sum_{j=1}^{m} \tilde{\beta}_{j m}(t) w_{1 j}\right|^{2}+\left|\tilde{\beta}_{j m}(t) w_{2 j}\right|^{2}\right) d x \\
& +(i r+\beta)|\Omega| \tilde{\beta}_{j m}(t)-\int_{\Omega} f_{1}(x) \bar{w}_{1 i} d x
\end{aligned}
$$

and

$$
\begin{aligned}
& h(\theta(t))-h(\tilde{\theta}(t)) \\
& =\alpha\left(\sum_{j=1}^{m} \beta_{j m}(t)-\sum_{j=1}^{m} \tilde{\beta}_{j m}(t)\right) \int_{\Omega}(-\Delta)^{\frac{s}{2}} w_{1 m}(-\Delta)^{\frac{s}{2}} \bar{w}_{1 i} d x \\
& \quad+\left(\beta_{j m}(t)-\tilde{\beta}_{j m}(t)\right) \int_{\Omega} g\left(\left|U_{m}\right|^{2}\right) d x \\
& \quad+(i r+\beta)|\Omega|\left(\beta_{j m}(t)-\tilde{\beta}_{j m}(t)\right) .
\end{aligned}
$$

Because of

$$
|\theta(t)-\tilde{\theta}(t)|=\sum_{j=1}^{m}\left|\beta_{j m}-\tilde{\beta}_{j m}\right|,
$$

and

$$
\begin{aligned}
& |\theta(t)|=\sqrt{\beta_{1 m}^{2}+\beta_{2 m}^{2}+\cdots+\beta_{m m}^{2}} \\
& |\tilde{\theta}(t)|=\sqrt{\tilde{\beta}_{1 m}^{2}+\tilde{\beta}_{2 m}^{2}+\cdots+\tilde{\beta}_{m m}^{2}} \\
& |h(\theta(t)-h(\tilde{\theta}(t)))| \\
& \leq \alpha\left|\sum_{j=1}^{m} \beta_{j m}(t)-\sum_{j=1}^{m} \tilde{\beta}_{j m}(t)\right|\left|\int_{\Omega}(-\Delta)^{\frac{s}{2}} w_{1 m}(-\Delta)^{\frac{s}{2}} \bar{w}_{1 i} d x\right| \\
& +\left|\beta_{j m}(t)-\tilde{\beta}_{j m}(t)\right| \int_{\Omega} g\left(\left|U_{m}\right|^{2}\right) d x+(r+\beta)|\Omega|\left|\beta_{j m}(t)-\tilde{\beta}_{j m}(t)\right| \\
& \leq \alpha \sum_{j=1}^{m}\left|\beta_{j m}-\tilde{\beta}_{j m}\right|\left|\int_{\Omega}(-\Delta)^{\frac{s}{2}} w_{1 m}(-\Delta)^{\frac{s}{2}} \bar{w}_{1 i} d x\right| \\
& +\sum_{j=1}^{m}\left|\beta_{j m}-\tilde{\beta}_{j m}\right| \int_{\Omega} g\left(\left|U_{m}\right|^{2}\right) d x+(r+\beta)|\Omega| \sum_{j=1}^{m}\left|\beta_{j m}-\tilde{\beta}_{j m}\right| \\
& =|\theta-\tilde{\theta}|\left(\alpha\left|\int_{\Omega}(-\Delta)^{\frac{s}{2}} w_{1 m}(-\Delta)^{\frac{s}{2}} \bar{w}_{1 i} d x\right|+\int_{\Omega} g\left(\left|U_{m}\right|^{2}\right) d x+(r+\beta)|\Omega|\right) .
\end{aligned}
$$


If $\int_{\Omega} g\left(\left|U_{m}\right|^{2}\right) d x \leq M,\left\{w_{j}(x)\right\}_{j=1}^{\infty} \in V$, because of $w_{1 j}, w_{1 i}, w_{2 j}, w_{2 i} \in V$, and $V \rightarrow H$ is compact, $\left\|w_{i j}\right\|_{V}^{2}$ is bounded and $\left\|w_{i j}\right\|_{H}^{2}$ also is bounded, then

$$
\alpha\left|\int_{\Omega}(-\Delta)^{\frac{s}{2}} w_{1 m} \sum_{i=1}^{m}(-\Delta)^{\frac{s}{2}} \bar{w}_{1 i} d x\right| \leq N_{1}
$$

and

$$
\frac{3}{2} \sum_{j=1}^{m} \int_{\Omega}\left|w_{1 j}\right|^{2}+\left|w_{2 j}\right|^{2} d x \leq N_{2}
$$

leads to

$$
|h(\theta)-h(\tilde{\theta})| \leq\left(N_{1}+C^{\prime} N_{2}+r|\Omega|+\beta|\Omega|\right)|\theta-\tilde{\theta}| .
$$

We finally get $h(\theta(t))$ is a Lipschitz continuous function and know that the ordinary differential equations (7.2) have common solutions for the unknown functions $\beta_{j m}(t)$, $j=1,2, \ldots, m$.

Theorem 7.1 For the given functions $F, U_{0}$,

$$
F \in H(\Omega), \quad U_{0} \in H(\Omega) \cap V(\Omega) .
$$

If $g(s) \geq 0, G(s)=\int_{0}^{s} g(s) d s \leq g(s) s$ and $\left|g^{\prime}(s)\right| \leq c_{0} s(s \geq 0)$, where $c>0$, then there exists a unique solution $U(x, t)$ for problem (1.10)-(1.11), and it satisfies the condition

$$
U \in L^{\infty}(0, T ; H(\Omega) \cap V(\Omega))
$$

Proof Under the condition above in this section, we continue to get the existence of the solution of problem (1.10)-(1.11). Firstly, we multiply (7.2) by $\beta_{j m}(t)$ and make sum about $j$,

$$
\begin{aligned}
& i\left(U_{m}^{\prime}, U_{m}\right)+\alpha\left((-\Delta)^{s} U_{m}, U_{m}\right)+\left(g\left(\left|U_{m}\right|^{2}\right) U_{m}, U_{m}\right)+i\left(Q U_{m}, U_{m}\right)+\left(\beta U_{m}, U_{m}\right) \\
& \quad=\left(F, \bar{U}_{m}\right) .
\end{aligned}
$$

It is similar to the process of (6.2), (6.4), from (6.5), we obtain

$$
\frac{d}{d t}\left\|U_{m}\right\|_{H}^{2}+\gamma\left\|U_{m}\right\|_{H}^{2} \leq \frac{1}{\gamma}\|F\|_{H}^{2}
$$

because of $F \in H(\Omega)$, $\|F\|_{H}^{2} \leq M_{1}$, and $U_{0 m} \in H(\Omega) \cap V(\Omega)$. For condition (7.3), by using Gronwall's inequality, we have a conclusion similar to (6.1)

$$
\left\|U_{m}(t)\right\|_{H}^{2} \leq\left\|U_{m}(x, 0)\right\|_{H}^{2} e^{-\gamma t}+\frac{\|F\|_{H}^{2}}{\gamma}\left(1-e^{-\gamma t}\right)
$$

then

$$
\limsup _{t \rightarrow \infty}\left\|U_{m}\right\|_{H}^{2} \leq \frac{M_{1}}{\gamma^{2}} .
$$

Therefore $U_{m}(t)$ is bounded in $H$. 
Secondly, we choose $w_{j}^{\prime}$ instead of $w_{j}$ in (7.2), then multiply (7.2) by $\bar{\beta}_{j m}(t)$, to make sum about $j$ :

$$
\begin{aligned}
& i\left(U_{m}^{\prime}, U_{m}^{\prime}\right)+\alpha\left((-\Delta)^{s} U_{m}, U_{m}^{\prime}\right)+\left(g\left(\left|U_{m}\right|^{2}\right) U_{m}, U_{m}^{\prime}\right)+i\left(Q U_{m}, U_{m}^{\prime}\right)+\beta\left(U_{m}, U_{m}^{\prime}\right) \\
& \quad=\left(F, U_{m}^{\prime}\right)
\end{aligned}
$$

and the real part of the equation above is

$$
\begin{aligned}
& \operatorname{Re} \alpha\left((-\Delta)^{\frac{s}{2}} U_{m},(-\Delta)^{\frac{s}{2}} \bar{U}_{m}^{\prime}\right)+\operatorname{Re} \int_{\Omega} g\left(\left|U_{m}\right|^{2}\right) U_{m} \bar{U}_{m}^{\prime} d x \\
& \quad-\operatorname{Im} \int_{\Omega} r u_{1 m} \bar{u}_{1 m}^{\prime}+\sigma u_{2 m} \bar{u}_{2 m}^{\prime} d x+\operatorname{Re} \beta\left(U_{m}, U_{m}^{\prime}\right) \\
& =\operatorname{Re}\left(F, U_{m}^{\prime}\right) .
\end{aligned}
$$

Return to see the real part of (7.9),

$$
\operatorname{Im}\left(U_{m}, U_{m}^{\prime}\right)+\left\|(-\Delta)^{\frac{s}{2}} U_{m}\right\|_{H}^{2}+\int_{\Omega} g\left(\left|U_{m}\right|^{2}\right)\left|U_{m}\right|^{2} d x+\beta\left\|U_{m}\right\|_{H}^{2}=\operatorname{Re}\left(F, U_{m}\right),
$$

where $G\left(\left|U_{m}\right|^{2}\right)=\int_{0}^{\left|U_{m}\right|^{2}} g(s) d s$. Combining (7.10) with (7.11), we finally get

$$
\begin{aligned}
& \frac{1}{2} \frac{d}{d t}\left\{\alpha\left\|(-\Delta)^{\frac{s}{2}} U_{m}\right\|_{H}^{2}+\int_{\Omega} G\left(\left|U_{m}\right|^{2}\right) d x+\beta\left\|U_{m}\right\|_{H}^{2}-2 \operatorname{Re}\left(F, U_{m}\right)\right\} \\
& \quad+\gamma\left\{\alpha\left\|(-\Delta)^{\frac{s}{2}} U_{m}\right\|_{H}^{2}+\int_{\Omega} G\left(\left|U_{m}\right|^{2}\right) d x+\beta\left\|U_{m}\right\|_{H}^{2}-2 \operatorname{Re}\left(F, U_{m}\right)\right\} \\
& \leq \operatorname{Re} \int_{\Omega}(r-2 \gamma) f_{1} \bar{u}_{1 m}+(\sigma-2 \gamma) f_{2} \bar{u}_{2 m} d x
\end{aligned}
$$

Introducing a functional equation about (7.12)

$$
\eta\left(U_{m}\right)=\beta\left\|U_{m}\right\|_{H}^{2}+\alpha\left\|(-\Delta)^{\frac{s}{2}} U_{m}\right\|_{H}^{2}+\int_{\Omega} G\left(\left|U_{m}\right|^{2}\right) d x-2 \operatorname{Re}\left(F, U_{m}\right)
$$

and rewriting (7.12) as

$$
\frac{1}{2} \frac{d}{d t} \eta\left(U_{m}\right)+\delta \eta\left(U_{m}\right) \leq \frac{1}{2}(\delta+2 \gamma)\left(\left\|U_{m}\right\|_{H}^{2}+\|F\|_{H}^{2}\right),
$$

we have

$$
\frac{d}{d t} \eta(U)+2 \gamma \eta(U) \leq 2 \gamma C_{\infty}^{\prime} .
$$

Finally, we can get

$$
\limsup _{t \rightarrow \infty} \eta\left(U_{m}\right) \leq C_{\infty}^{\prime}
$$

and

$$
\beta\left\|U_{m}\right\|_{H}^{2}+\alpha\left\|(-\Delta)^{\frac{s}{2}} U_{m}\right\|_{H}^{2}+\int_{\Omega} G\left(\left|U_{m}\right|^{2}\right) d x \leq C_{\infty}^{\prime}+\frac{1}{2}\left(M_{1}+\frac{M_{1}}{\gamma^{2}}\right) .
$$


Because of $\beta\left\|U_{m}\right\|_{H}^{2} \geq 0, \int_{\Omega} G\left(\left|U_{m}\right|^{2}\right) d x \geq 0$, we know that $\left\|(-\Delta)^{\frac{s}{2}} U_{m}\right\|_{H}^{2}=\left\|U_{m}\right\|_{V}^{2}$, and $U_{m}(t)$ is bounded in $V$.

Hence from the sequence $\left\{U_{m}(x, t)\right\}$ of approximate solutions, we can select a subsequence $\left\{U_{\mu}(x, t)\right\}$ and have a function $U(x, t) \in L^{\infty}(0, T ; H)$ such that

$$
\begin{aligned}
& U_{\mu}(x, t) \rightarrow U(x, t) \quad \text { in } U(x, t) \in L^{\infty}(0, T ; H) \text { weakly star, } \mu \rightarrow \infty . \\
& (-\Delta)^{\frac{s}{2}} U_{\mu}(x, t) \rightarrow(-\Delta)^{\frac{s}{2}} U(x, t) \quad \text { in } U(x, t) \in L^{\infty}(0, T ; V) \text { weakly star and a.e., } \mu \rightarrow \infty . \\
& g\left(\left|U_{\mu}\right|^{2}\right)\left|U_{\mu}\right|^{2} \rightarrow g\left(|U|^{2}\right)|U|^{2} \quad \text { in } U(x, t) \in L^{\infty}\left(0, T ; L^{6}(\Omega)\right) \text { weakly star, } \mu \rightarrow \infty .
\end{aligned}
$$

And

$$
\begin{aligned}
& U_{\mu}(x, t) \rightarrow U(x, t) \quad \text { in } U(x, t) \in L^{2}(0, T ; H) \quad \text { weakly, } \mu \rightarrow \infty . \\
& (-\Delta)^{\frac{s}{2}} U_{\mu}(x, t) \rightarrow(-\Delta)^{\frac{s}{2}} U(x, t) \quad \text { in } U(x, t) \in L^{2}(0, T ; V) \text { weakly and a.e., } \mu \rightarrow \infty . \\
& g\left(\left|U_{\mu}\right|^{2}\right)\left|U_{\mu}\right|^{2} \rightarrow g\left(|U|^{2}\right)|U|^{2} \quad \text { in } U(x, t) \in L^{2}\left(0, T ; L^{6}(\Omega)\right) \text { weakly, } \mu \rightarrow \infty .
\end{aligned}
$$

From

$$
\left(i U_{\mu}^{\prime}-\alpha(-\Delta)^{s} U_{\mu}+G_{\mu} U_{\mu}+i Q U_{\mu}+\beta U_{\mu}-F, U_{\mu}\right)=0,
$$

hence the function $U(x, t)$ satisfies equation (1.11) everywhere and the boundary initial conditions (1.10). So the existence of solution for problem (1.10)-(1.11) has been proved.

\section{The uniqueness of solution}

Let $U_{1}, U_{2}$ be solutions of (1.11) satisfying the conditions of Theorem 7.1. We have $W=$ $U_{1}-U_{2}$ and $W(0)=0$, we have

$$
\begin{array}{ll}
U_{2}=\left(u_{21}, u_{22}\right)^{T}, & \left|U_{2}\right|^{2}=\left|u_{21}\right|^{2}+\left|u_{22}\right|^{2} ; \\
U_{1}=\left(u_{11}, u_{12}\right)^{T}, & \left|U_{1}\right|^{2}=\left|u_{11}\right|^{2}+\left|u_{12}\right|^{2} .
\end{array}
$$

Then we obtain

$$
i w_{t}+\alpha(-\Delta)^{s} W+i Q w+\beta W=g\left(\left|U_{2}\right|^{2}\right) U_{2}-g\left(\left|U_{1}\right|^{2}\right) U_{1}
$$

Making a scalar product with (8.1) by vector $W$ over $\Omega$, then

$$
\begin{aligned}
& i\left(W_{t}, W\right)+\alpha\left((-\Delta)^{s} W, W\right)+i(Q w, W)+\beta(W, W) \\
& \quad=\left(g\left(\left|U_{2}\right|^{2}\right) U_{2}-g\left(\left|U_{1}\right|^{2}\right) U_{1}, W\right) .
\end{aligned}
$$

Choose the imaginary part of (8.2)

$$
\frac{1}{2} \frac{d}{d t}\|W\|_{H}^{2}+\int_{\Omega} r\left|W_{1}\right|^{2}+\sigma\left|W_{2}\right|^{2} d x=\operatorname{Im} \int_{\Omega}\left(g\left(\left|U_{2}\right|^{2}\right) U_{2}-g\left(\left|U_{1}\right|^{2}\right) U_{1}\right) \bar{W} d x .
$$


Set $\gamma=\min (r, \sigma)$,

$$
\frac{1}{2} \frac{d}{d t}\|W\|_{H}^{2}+\gamma\|W\|_{H}^{2} \leq \operatorname{Im} \int_{\Omega}\left(g\left(\left|U_{2}\right|^{2}\right) U_{2}-g\left(\left|U_{1}\right|^{2}\right) U_{1}\right) \bar{W} d x
$$

Firstly we shall prove

$$
\begin{aligned}
& \left|g\left(\left|U_{2}\right|^{2}\right) U_{2}-g\left(\left|U_{1}\right|^{2}\right) U_{1}\right| \\
& \quad \leq\left|g\left(\left|U_{2}\right|^{2}\right)\left(U_{2}-U_{1}\right)+\left(g\left(\left|U_{2}\right|^{2}\right)-g\left(\left|U_{1}\right|^{2}\right)\right) U_{1}\right| \\
& \quad \leq g\left(\left|U_{2}\right|^{2}\right)\left|U_{2}-U_{1}\right|+\left|g^{\prime}\left(\theta\left|U_{1}\right|^{2}+(1-\theta)\left|U_{2}\right|^{2}\right)\right|\left|U_{1}\right|\left|U_{2}-U_{1}\right| \\
& \quad=\left(g\left(\left|U_{2}\right|^{2}\right)+\left|U_{1}\right| g^{\prime}\left(\theta\left|U_{1}\right|^{2}+(1-\theta)\left|U_{2}\right|^{2}\right)\right)\left|U_{2}-U_{1}\right|,
\end{aligned}
$$

where $0 \leq \theta \leq 1$. Since $\Omega \subset R^{2}, H_{0}^{1}(\Omega)$ is embedding to $L^{\infty}(\Omega)$, and

$$
\|U\|_{L^{\infty}(\Omega) \times L^{\infty}(\Omega)}^{2} \leq C\|U\|_{V}^{2}
$$

by using Hölder's inequality of the form

$$
\int_{\Omega}\left|U_{1}\right|\left|g^{\prime}\right| d x \leq\left\|U_{1}\right\|_{L^{\infty}(\Omega) \times L^{\infty}(\Omega)}\left\|g^{\prime}\right\|_{L^{1}(\Omega) \times L^{1}(\Omega)},
$$

we get the deduce of (8.3)

$$
\begin{aligned}
& \frac{1}{2} \frac{d}{d t}\|W\|_{H}^{2}+\gamma\|W\|_{H}^{2} \\
& \quad \leq \int_{\Omega}\left|g\left(\left|U_{2}\right|^{2}\right) U_{2}-g\left(\left|U_{1}\right|^{2}\right) U_{1}\right||\bar{W}| d x \\
& \quad \leq \int_{\Omega}\left(g\left(\left|U_{2}\right|^{2}\right)+\left|U_{1}\right| g^{\prime}\left(\theta\left|U_{1}\right|^{2}+(1-\theta)\left|U_{2}\right|^{2}\right)\right)\left|U_{2}-U_{1}\right||\bar{W}| d x \\
& \quad \leq\left(1+\frac{c_{0}}{2}\right) \int_{\Omega}\left(\left|U_{2}\right|^{2}+\left|U_{1}\right|^{2}\right)|W|^{2} d x \\
& \quad \leq\left(1+\frac{c_{0}}{2}\right)\left(\left\|U_{2}\right\|_{L^{\infty}(\Omega) \times L^{\infty}(\Omega)}^{2}+\left\|U_{1}\right\|_{L^{\infty}(\Omega) \times L^{\infty}(\Omega)}^{2}\right) \int_{\Omega}|W|^{2} d x \\
& \quad \leq \frac{3 \tilde{c}}{2}\left(\left\|U_{2}\right\|_{V}^{2}+\left\|U_{1}\right\|_{V}^{2}\right)\|W\|_{H}^{2} \cdot
\end{aligned}
$$

From Sect. 6, we know that $\left\|U_{1}\right\|_{V}^{2}+\left\|U_{2}\right\|_{V}^{2}$ is bounded. Rewrite (8.4) as follows:

$$
\frac{1}{2} \frac{d}{d t}\|W\|_{H}^{2}+\left(\gamma-\frac{3 \tilde{c}}{2}\left\|U_{2}\right\|_{V}^{2}-\frac{3 \tilde{c}}{2}\left\|U_{1}\right\|_{V}^{2}\right)\|W\|_{H}^{2} \leq 0
$$

Because of $W(0)=0$, we finally get $W=0$.

\section{The global attractor}

Furthermore, for every $t \geq 0$, the mapping

$$
S(t): U_{0} \rightarrow U(t)
$$


is continuous and bounded in $H$ and $V$. It follows from the uniqueness of solution (1.10)(1.11) that the family $\{S(t)\}_{t \geq 0}$ forms a semi-group:

$$
S\left(t_{1}+t_{2}\right)=S\left(t_{1}\right) S\left(t_{2}\right), \quad t_{1}, t_{2} \geq 0, \quad S(0)=I .
$$

Another important property is that the semi-group $S(t)$ is compact on $H$ for $t>0$. That is to say the image of $S(t)$ of any bounded set in $H$ is relatively compact in $H$. In order to prove the existence of global attractors of problem (1.10)-(1.11), we need the following result.

\subsection{Absorbing ball in $H$}

From Sect. 7,

$$
\|U(t)\|_{H}^{2} \leq\left\|U_{0}\right\|_{H}^{2} e^{-\gamma t}+\frac{\|F\|_{H}^{2}}{\gamma}\left(1-e^{-\gamma t}\right), \quad t \in(0, T),
$$

and

$$
\limsup _{t \rightarrow \infty}\|U\|_{H}^{2} \leq \rho_{0}^{2}, \quad \rho_{0}^{2}=\frac{M_{1}}{\gamma^{2}} .
$$

We know that $B_{0}=B_{H}\left(0, \rho_{0}\right)$ is the absorbing set in $H$ for the semi-group $S(t)$, and

$$
\rho_{0}^{2} \geq \frac{M_{1}}{\gamma}
$$

We infer from (9.2) that the balls $B_{0}=B_{H}\left(0, \rho_{0}\right)$ of $H$ with $\rho \geq \rho_{0}$ are positive invariants for the semigroup $S(t)$, and these balls are absorbing for any $\rho>\rho_{0}$. We choose $\rho_{0}^{\prime}>\rho_{0}$ and denote by $B_{0}$ the ball $B_{H}\left(0, \rho_{0}^{\prime}\right)$. And the set $B$ bounded in $H$ is included in a ball $B(0, R)$ of $H$. It is easy to deduce from (9.2) that $S(t) B \subset B_{0}$ for $t \geq t_{0}\left(B, \rho_{0}^{\prime}\right)$, where

$$
t_{0}=\frac{1}{\gamma} \log \frac{\gamma^{2} R^{2}}{\gamma^{2} \rho_{0}^{2}-c_{1}} .
$$

We infer from (6.5) that integration in $t, \tau>0$ yields

$$
\gamma \int_{t}^{t+\tau}\|U\|_{H}^{2} d s \leq\|U\|_{H}^{2}+\frac{M_{1} \tau}{\gamma}
$$

With the use of (9.3) we conclude that

$$
\limsup _{t \rightarrow \infty} \int_{t}^{t+\tau}\|U\|_{H}^{2} d s \leq \frac{M_{1} \tau}{\gamma^{3}}+\frac{M_{1} \tau}{\gamma^{2}} .
$$

In Sect. 7, we get the inequality

$$
\frac{d}{d t} \eta(U)+2 \gamma \eta(U) \leq 2 \gamma C_{\infty}
$$

and

$$
\limsup _{t \rightarrow \infty}\left(\beta\|U\|_{H}^{2}+\alpha\left\|(-\Delta)^{\frac{s}{2}} U\right\|_{H}^{2}+\int_{\Omega} G\left(|U|^{2}\right) d x-2 \operatorname{Re}(F, U)\right) \leq C_{\infty} .
$$


Integrating (9.7) between $t$ and $t+\tau$

$$
\begin{aligned}
& \limsup _{t \rightarrow \infty}\left\{\int_{t}^{t+\tau} \beta\|U(s)\|_{H}^{2}+\alpha\left\|(-\Delta)^{\frac{s}{2}} U(s)\right\|_{H}^{2}+\|U(s)\|_{L^{4}(\Omega)}^{4}-2 \operatorname{Re}(F, U(s)) d s\right. \\
&\left.\quad+\beta\|U(t+\tau)\|_{H}^{2}+\alpha\left\|(-\Delta)^{\frac{s}{2}} U(t+\tau)\right\|_{H}^{2}+\|U(t+\tau)\|_{L^{4}(\Omega)}^{4}-2 \operatorname{Re}(F, U(t+\tau))\right\} \\
& \quad \leq 2 \gamma C_{\infty}(1+\tau),
\end{aligned}
$$

we obtain

$$
\limsup _{t \rightarrow \infty} \int_{t}^{t+\tau}\left(\beta\|U(s)\|_{H}^{2}+\alpha\left\|(-\Delta)^{\frac{s}{2}} U(s)\right\|_{H}^{2}+\int_{\Omega} G\left(|U|^{2}\right) d x\right) d s \leq C_{s} .
$$

\subsection{Absorbing ball in $V$}

We continue and show the existence of an absorbing set in $V$ and the uniform compactness of $S(t)$. For that purpose, we know that

$$
\eta(U)=\beta\|U\|_{H}^{2}+\alpha\left\|(-\Delta)^{\frac{s}{2}} U\right\|_{H}^{2}+\int_{\Omega} G\left(|U|^{2}\right) d x-2 \operatorname{Re}(F, U) .
$$

If $F \in H \cap V$, we have

$$
\eta(U) \leq \eta\left(U_{0}\right) e^{-2 \gamma t}+C_{\infty}\left(1-e^{-2 \gamma t}\right)
$$

then

$$
\limsup _{t \rightarrow \infty}\left(\beta\|U\|_{H}^{2}+\alpha\left\|(-\Delta)^{\frac{s}{2}} U\right\|_{H}^{2}+\int_{\Omega} G\left(|U|^{2}\right) d x-2 \operatorname{Re}(F, U)\right) \leq C_{\infty}
$$

and $\lim \sup _{t \rightarrow \infty} \alpha\left\|(-\Delta)^{\frac{s}{2}} U\right\|_{H}^{2} \leq \rho_{1}^{2}, \rho_{1}=C_{\infty}$.

We recall that $\left(\|U\|^{2}+\left\|(-\Delta)^{\frac{s}{2}} U\right\|^{2}\right)^{1 / 2}$ is the norm on $V\left(=\right.$ the $H^{1}(\Omega)$ norm). Combining (9.5), we obtain the existence of an absorbing set in $V$ for $S(t)$ and uniform compactness property:

The operator $S(t)$ is uniformly compact for $t$ large. By this we mean that for every bounded set $B$ there exists $t_{0}$, which may depend on $B$, such that

$$
\bigcup_{t \geq t_{0}} S(t) B
$$

is relatively compact in $H$.

Indeed, if $B$ is a bounded set of $V$, then it is also a bounded set of $H$,

$$
S(t) B \subset B_{0} \quad \text { for } t \geq t_{0}\left(B, B_{0}\right)
$$

and then with (9.3)

$$
S(t) B \subset B_{1} \quad \text { for } t \geq t_{0}+\tau,
$$

where $B_{1}$ is the ball of $V$ centered at 0 of radius $\rho_{1}>\rho_{0}$. 
The ball of $V, B_{1}=B_{V}\left(0, \rho_{1}\right)$ centered at 0 of radius $\rho_{1}$ is absorbing in $V$ for the semigroup $S(t)$.

If $U_{0} \in B$, where $B$ is only bounded in $H$, the above analysis still applies and

$$
S(t) B \subset B_{1} \quad \text { for } t \geq t_{0}(B)+\tau .
$$

Since $B_{1}$ is bounded in $V$ and the injection of $V$ in $H$ is compact, we conclude that $\bigcup_{t \geq t_{0}+\tau} S(t) B$ is relatively compact in $H$.

Definition 9.1 The $\omega$-limit set $\mathrm{K}$ of $B$

$$
\mathrm{K}=\bigcap_{\tau>0} \overline{\bigcup_{t>t_{0}+\tau} S(t) B}
$$

where the closure is taken in $H$.

Therefore, from the a priori estimates, using Theorem I.1.1 in [32], the $\omega$-limit set of $B, \mathrm{~K}=\omega(B)$, is a global attractor. It is an infinite-dimensional dynamic system associated with this evolution equation (1.11) supplemented by the Dirichlet boundary condition. $\mathrm{K}$ attracts the bounded sets of $H$. This dynamic system possesses an attractor $\mathrm{K}$ which is compact in $H$.

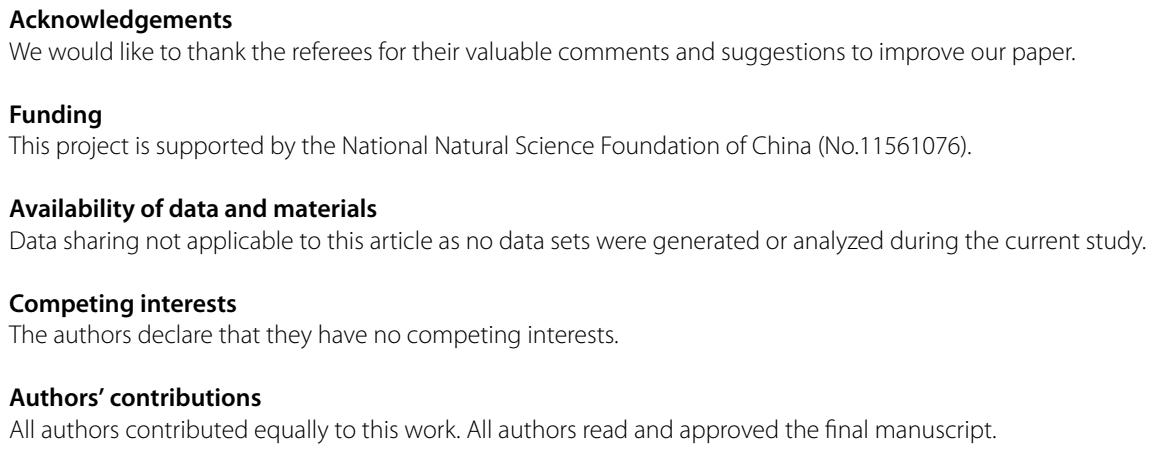

\section{Publisher's Note}

Springer Nature remains neutral with regard to jurisdictional claims in published maps and institutional affiliations.

Received: 1 April 2018 Accepted: 13 June 2018 Published online: 11 July 2018

\section{References}

1. Kaminow, I.P.: Polarization in optical fibers. IEEE J. Quantum Electron. 17, 15-22 (1981)

2. Menyuk, C.R.: Nonlinear pulse propagation in birefringence optical fiber. IEEE J. Quantum Electron. 23, 174-176 (1987)

3. Menyuk, C.R.: Pulse propagation in an elliptically birefringent Kerrmedium. IEEE J. Quantum Electron. 25, 2674-2682 (1989)

4. Hasegawa, A., Kodama, Y.: Solitons in Optical Communications. Academic Press, San Diego (1995)

5. Islam, M.N.: Ultrafast Fiber Switching Devices and Systems. Cambridge Univ. Press, New York (1992)

6. Menyuk, C.R.: Stability of solitons in birefringent optical Fibers. II. Arbitrary Amplitudes. J. Opt. Soc. Am. B 5, 392-402 (1998)

7. Yang, J.: Multisoliton perturbation theory for the Manakov equations and its applications to nonlinear optics. Phys. Rev. E 59(2), 2393-2405 (1999)

8. Berezin, F.A., Shubin, M.A.: The Schrödinger Equation. Kluwer Academic, Dordrecht (1983) Published by the Moscow State University

9. Wadati, M., Izuka, T., Hisakado, M.: A coupled nonlinear Schrödinger equation and optical solitons. J. Phys. Soc. Jpn. $61(7), 2241-2245$ (1992) 
10. Guo, B., Su, F.Q.: The global attractors for the periodic initial value problem of generalized Kuramato-Sivashinsky type equations in multi-dimensions. J. Partial Differ. Equ. 6, 217-236 (1993)

11. Christiansen, P.L., Eilbeck, J.C., Enolskii, V.Z., Kostov, N.A.: Quasi-periodic and periodic solutions for coupled nonlinear Schrödinger equations of Manakov type. Proc. R. Soc. Lond. A 456, 2263-2281 (2001). https://doi.org/10.1098/rspa.2000.0612

12. Sulem, C., Sulem, P.-L.: The Nonlinear Schrödinger Equation: Self-Focusing and Wave Collapse. Applied Mathematical Sciences, vol. 139. Springer, New York (1999). https://doi.org/10.1007/b98958

13. Sun, J.Q., Gu, X.Y., Ma, Z.Q.: Numerical study of the soliton waves of the coupled nonlinear Schrödinger system. Physica D 196, 311-328 (2004)

14. Zhu, C.S.: Attractor for the nonlinear Schrödinger equation with harmonic potential. J. Southwest China Normal Univ. (Nat. Sci.) 30(5), 788-791 (2005)

15. Utsumi, T., Aoki, T., Koga, J., Yamagiwa, M.: Solutions of the 1D coupled nonlinear Schrödinger equations by the CIP-BS method. Commun. Comput. Phys. 1, 261-275 (2006)

16. Pomponio, A.: Coupled nonlinear Schrödinger systems with potentials. J. Differ. Equ. 227, 258-281 (2006)

17. Warren, O.H., Elgin, J.N.: The vector nonlinear Schrödinger hierarchy. Physica D 228, 166-171 (2007)

18. Ismai, M.S.: Numerical solution of coupled nonlinear Schrödinger equation by Galerkin method. Math. Comput. Simul. 78, 532-547 (2008)

19. Goubet, O., Molinet, L.: Global attractor for weakly damped nonlinear Schrödinger equations in $L^{2}(R)$. Nonlinear Anal. 71, 317-320 (2009)

20. Zhong, P.H., Yang, R.H., Yang, G.S.: Exact periodic and blow up solutions for 2D Ginzburg-Landau equation. Phys. Lett. A 373(1), 19-22 (2008). https://doi.org/10.1016/j.physleta.2008.10.078

21. Guo, B.L., Yang, G.S.: Some exact nontrivial global solutions with values in unit sphere for two-dimensional Landau-Lifshitz equations. J. Math. Phys. 42(11), 5223-5227 (2001)

22. Yang, G.S.: The difference between Schrödinger equation derived from Schrödinger map and Landau-Lifshitz equation. Phys. Lett. A 376(4), 231-235 (2012). https://doi.org/10.1016/j.physleta.2011.11.003

23. Yang, G.S., Guo, B.L.: Some exact solutions to multidimensional Landau-Lifshitz equation with uprush external field and anisotropy field. Nonlinear Anal., Theory Methods Appl. 71(9), 3999-4006 (2009). https://doi.org/10.1016/j.na.2009.02.070

24. Yang, G.S., Liu, X.G.: Spherical cone symmetric families generated by Landau-Lifshitz equation and their evolution. Sci. China Math. 41(2), 181-196 (2011)

25. Caffarelli, L., Silvestre, L.: An extension problem related to the fractional Laplacian. Commun. Partial Differ. Equ. 32 1245-1260 (2007)

26. Barrios, B., Colorado, E., de Pablo, A., Sánchez, U.: On some critical problems for the fractional Laplacian operator. J. Differ. Equ. 252(11), 6133-6162 (2012). https://doi.org/10.1016/j.jde.2012.02.023

27. Abe, S. Thurner, S.: Anomalous diffusion in view of Einstein's 1905 theory of Brownian motion. Physica A 356 403-407 (2005)

28. Jara, M.: Nonequilibrium scaling limit for a tagged particle in the simple exclusion process with long jumps. Commun. Pure Appl. Math. 62, 198-214 (2009)

29. Vlahos, L., Isliker, H., Kominis, Y., Hizonidis, K.: Normal and anomalous diffusion: a tutorial. In: Bountis, T. (ed.) Order and Chaos, vol. 10. Patras University Press, Patras (2008)

30. Evans, L.C.: Partial Differential Equations, 2nd edn. Graduate Studies in Mathematics, vol. 19. Am. Math. Soc., Providence (1998)

31. Kilbas, A.A., Srivastava, H.M., Trujillo, J.J.: Theory and Applications of Fractional Differential Equations. North-Holland Mathematics Studies, vol. 204. Elsevier, Amsterdam (2006)

32. Teman, R.: Infinite-Dimensional Dynamical Systems in Mechanics and Physics. Applied Mathematical Sciences, vol. 68. Springer, New York (1988). https://doi.org/10.1007/978-1-4684-0313-8

\section{Submit your manuscript to a SpringerOpen ${ }^{\circ}$ journal and benefit from:}

- Convenient online submission

- Rigorous peer review

- Open access: articles freely available online

- High visibility within the field

- Retaining the copyright to your article

Submit your next manuscript at $\gg$ springeropen.com 